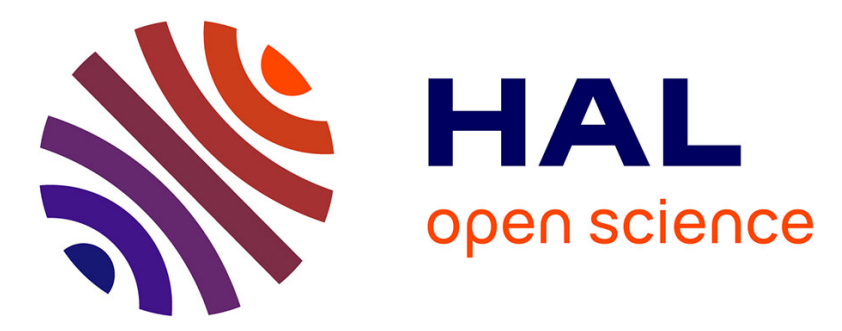

\title{
Role of interlayer porosity and particle organization in the diffusion of water in swelling clays
}

\author{
A. Asaad, F. Hubert, E. Ferrage, T. Dabat, E. Paineau, P. Porion, S. Savoye, \\ B. Gregoire, B. Dazas, Alfred Delville, et al.
}

\section{- To cite this version:}

A. Asaad, F. Hubert, E. Ferrage, T. Dabat, E. Paineau, et al.. Role of interlayer porosity and particle organization in the diffusion of water in swelling clays. Applied Clay Science, 2021, 207, pp.106089. 10.1016/j.clay.2021.106089 . hal-03212181

\section{HAL Id: hal-03212181 \\ https://hal.science/hal-03212181}

Submitted on 29 Apr 2021

HAL is a multi-disciplinary open access archive for the deposit and dissemination of scientific research documents, whether they are published or not. The documents may come from teaching and research institutions in France or abroad, or from public or private research centers.
L'archive ouverte pluridisciplinaire HAL, est destinée au dépôt et à la diffusion de documents scientifiques de niveau recherche, publiés ou non, émanant des établissements d'enseignement et de recherche français ou étrangers, des laboratoires publics ou privés. 


\section{Role of interlayer porosity and particle organization in the diffusion of}

\section{water in swelling clays}

3

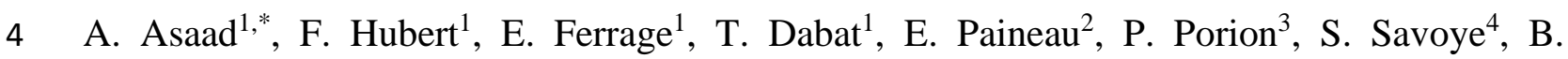

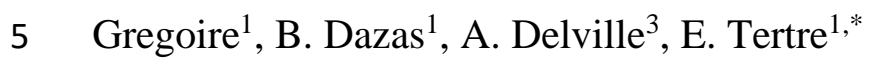

6

$7 \quad{ }^{1}$ IC2MP, Equipe HydrASA, UMR 7285 CNRS/Université de Poitiers, 86073 Poitiers, France.

$8 \quad{ }^{2}$ Université Paris-Saclay, CNRS, Laboratoire de Physique des Solides, 91405 Orsay, France

$9 \quad{ }^{3}$ ICMN, UMR 7374 CNRS/Université d'Orléans, 45071 Orléans, France

$10{ }^{4}$ Université Paris-Saclay, CEA, Service d'Etude du Comportement de Radionucléides, 91191

11 Gif-sur-Yvette, France

12

* E-mail addresses of the corresponding authors:

A. Asaad: ali.asaad@univ-poitiers.fr

E. Tertre: emmanuel.tertre@univ-poitiers.fr

\section{Highlights}

- Samples with same interparticle porosity but different particle organizations.

20 - Reduction in the overall mobility of water in presence of interlayer porosity.

- Minor role of interlayer porosity in flux traversing samples.

- Anisotropy in water diffusion quantitatively related to the preferential orientation of the particles. 

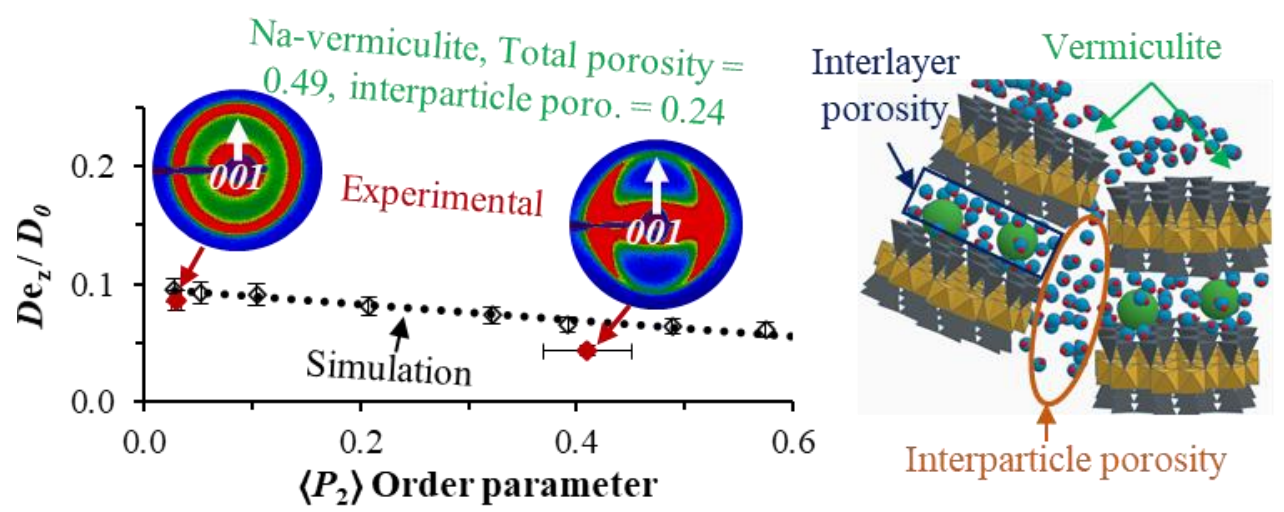

\section{Abstract}

This study focuses on the role played by the interparticle and interlayer porosities, and the preferred orientation of the particles on water diffusion in dual-porosity clayey media (i.e., swelling clay minerals). For this purpose, we use Na-vermiculite, a swelling clay that does not exhibit osmotic swelling and therefore allows a clear discrimination between the interparticle and interlayer porosities. Two samples were prepared with an equal proportion of interparticle and interlayer porosities (i.e., 0.25 each) but different degree of preferred particle orientation (i.e., isotropic vs anisotropic). Through-diffusion and pulsed gradient spin echo attenuation measurements by nuclear magnetic resonance of protons techniques were used to probe water mobility, while the orientation of the particles was quantified by X-ray scattering analysis. Experimental water diffusion results obtained with these two Na-vermiculite samples were compared to those with samples made of Na-kaolinite particles (non-swelling clay mineral) having only interparticle porosities equal to 0.25 and 0.5 , corresponding respectively to the interparticle and the total porosity of the Na-vermiculite samples. In addition, these experimental results were compared to simulated data using Brownian dynamics with virtual porous media representative of the real samples. For the range of porosities investigated, a good agreement was observed between measured and simulated water mobilities. The obtained results confirmed the important role played by the preferential orientation of the particles on water dynamics in clayey media, through an important reduction of overall water mobility 
between the isotropic and anisotropic Na-vermiculite samples. These results also showed that for the same total porosity, the presence of interlayer porosity and associated nano-confinement led to a logical reduction in the pore diffusion coefficient of water in Na-vermiculite in comparison to Na-kaolinite. Moreover, in comparison with Na-kaolinite having the same interparticle porosity, results showed that the contribution of the interlayer volume on the traversing flux was small compared to the interparticle volume. Finally, the computed results revealed that the anisotropy in water diffusion can be directly predicted based on the degree of particles preferred orientation, irrespective of the total or the distribution of the different porosity types.

\section{Introduction}

Diffusion is the main transport process in compacted clayey rocks owing to their low hydraulic conductivity (Pusch, 1979; Bourg et al., 2003; Bourg and Tournassat, 2015). This process has been extensively studied, especially in the context of the storage of nuclear waste and $\mathrm{CO}_{2}$, and resulted in an abundance of experimental data obtained by various methods (such as through-diffusion (TD) experiments using tracers or quasi-elastic neutron scattering) and models describing the diffusion of different elements in clayey media (e.g., Sato and Suzuki, 2003; Van Loon et al., 2004; Andra, 2005; García-Gutiérrez et al., 2006; Landais, 2006; Bachu, 2008; González Sánchez et al., 2008a and b; Glaus et al., 2010; Gimmi and Kosakowski, 2011; Altmann et al., 2015; Savoye et al., 2015; Charlet et al., 2017; Bestel et al., 2018; Dagnelie et al., 2018). Note that these data were obtained for various media constitutive of mono- or multiclayey phases and considering the presence or not of non-clayey minerals (Shackelford and Moore, 2013; Bourg and Tournassat, 2015; Charlet et al., 2017; Bestel et al., 2018 and references therein).

The organization and connectivity of the pore network are the main parameters that control diffusion of water in clayey media since water is usually inert from a chemical 
viewpoint (Bourg and Tournassat, 2015 and references therein). The influence of the total porosity value is the parameter that has been the subject of most extensive investigation. Thus, a logical decrease in the water diffusion coefficients with a diminution in the porosity is commonly reported (e.g., Tournassat and Steefel, 2019 and references therein). In contrast, less attention has been paid to the influence of other parameters, such as the preferential orientation of the clay particles, a typical property of these platelet materials in natural aqueous environments (Bennett et al., 1981), or to the distribution of different types of porosities (e.g., interlayer $v s$. interparticle porosity in swelling clay minerals). Nevertheless, by investigating the spatial anisotropy of the diffusion of water tracers in such media, either by TD experiments or by analyzing the 3D distribution of the tracer, several authors (Farver and Yund, 1999; Sato, 2000; Sato and Suzuki, 2003; Suzuki et al., 2004; García-Gutiérrez et al., 2006; Wenk et al., 2008; Wittebroodt et al., 2012; Xiang et al., 2013; Jacops et al., 2017; Dagnelie et al., 2017) have reported a significant diminution of the effective diffusion coefficients $(D e)$ of water in the direction perpendicular to the sedimentation beds with respect to the parallel direction. Such behavior was attributed to anisotropy in the shape and distribution of the pores between clay particles, resulting from their preferred orientation, as evidenced by either X-ray scattering (XRS, Wenk et al., 2008) or microscopy-imaging techniques (Sato, 2000; Suzuki et al., 2004).

The porosity and preferred orientation of the particles in clayey media are often coupled parameters (a decrease in the porosity leads to an increase in the particle orientation). Hence, it is complicated to discriminate the contribution of each parameter to the overall diffusion process. To overcome such limitation, Dabat et al., (2020) recently proposed a methodology that allow to prepare samples from Na-kaolinite particles (non-porous and non-charged particles) with similar porosity but different degrees of preferential orientation of the particles. These authors succeeded in connecting the anisotropy of the tensor diffusion of the water tracer to the preferential orientation of the particles in these samples for a porosity value of $\sim 0.5$ based 
on experimental water diffusion analyses obtained using pulsed gradient spin echo attenuation measurements by nuclear magnetic resonance (PGSE-NMR) and numerical data from Brownian dynamics (BD) simulations using various virtual porous media (VPMs). However, such a relationship remains to be investigated in the case of clay media made of swelling clay particles with two types of porosities (i.e., interlayer and interparticle) being accessible for the water. In such dual-porosity media with charged surfaces, water mobility in the interlayer space can be drastically reduced compared to that in the interparticle porosity, as revealed by molecular dynamic simulations (Titiloye and Skipper, 2001; Marry et al., 2002; Malikova et al., 2006; Michot et al., 2012 among others) and quasi-elastic neutron scattering studies (González Sánchez et al., 2008a; Bestel, 2014) performed on low charge swelling clays, e.g. montmorillonite. Note that this reduced mobility near the surface of the charged clayey particles is usually considered in reactive transport models (i.e., models that couple transport to chemical properties using continuum approaches (Ochs et al., 2001; Tournassat and Steefel, 2019 and references therein), which predict the diffusion of water in complex and heterogenous clayey media. The use of these numerical approaches allow for decoupling the diffusion that occurs in the different types of porosity (i.e., interlayer $v s$. interparticle porosities), while discrete models (e.g., molecular dynamics, random walk or BD simulations) also allow consideration of the specific role of the particles' preferred orientation on the diffusion properties (e.g., Churakov and Gimmi, 2011; Tyagi et al., 2013; Bacle et al., 2016; Gimmi and Churakov, 2019; Dabat et al., 2020; Underwood and Bourg, 2020). Beyond such numerical approaches, existing experimental water diffusion data in dual-porosity media were almost exclusively obtained with smectite particles (e.g., Bourg and Tournassat, 2015 and references therein). This mineral displays, however, osmotic swelling in water-saturated conditions (i.e., incorporation of an illdefined number of water molecules between the layers (Abend and Lagaly, 2000; Liu, 2013; Tertre et al., 2018 among others), which hampers the calculation of the respective interlayer 
and interparticle porosity volumes. In the scope of quantitatively discussing the role played by interlayer and interparticle porosities on the diffusion of water, as well as by the preferred orientation of the clay particles, additional experimental data on dual-porosity clay media that are not sensitive to osmotic swelling are required. For this purpose, vermiculite clay mineral represents an ideal candidate. Vermiculite is a swelling clay mineral with a structure similar to smectite. However, owing to the higher layer charge, this mineral does not display osmotic swelling (Faurel, 2012) and exhibits well-defined interlayer volumes in water-saturated conditions, which allows for a better determination of the different interlayer and interparticle volumes in the samples. Tertre et al. (2018) used this mineral to perform water tracer diffusion experiments and highlighted the notable differences in the effective diffusion coefficients obtained in vermiculite compared to montmorillonite for similar total porosities. Moreover, by comparison with experimental data reported for kaolinite, these authors noticed that the experimental data were similar for the same interparticle porosity value. However, because the preferred orientation of the clay particles was not measured, it was not possible to deduce information on the contribution of different parameters (the porosity distribution or particles' orientation) to the overall water diffusion process.

The present study represents an extension of the work of Tertre et al. (2018) and the recent approach proposed by Dabat et al. (2020) for kaolinite clay mineral (at a porosity value of $\sim 0.5$ ), to the dual-porosity media made of Na-vermiculite particles. In this work, an experimental methodology was developed to obtain vermiculite samples with two degrees of preferential particle orientation, as measured by XRS analysis, for the same total porosity of $50 \%$ (the same total porosity used by Dabat et al. (2020) for Na-kaolinite samples). In such conditions, Navermiculite is composed of an equal proportion of interlayer and interparticle porosity proportions (i.e., 0.25). Experimental diffusion data on water tracer (HDO) in these media were obtained using TD experiments that allow to determine the effective diffusion coefficients 
in the direction perpendicular to the sedimentation plane of the particles. To complete the experimental dataset on water diffusion, the sample set also included an additional clay medium of Na-kaolinite with a total porosity of $\sim 0.25$ (i.e., similar interparticle porosity as with Navermiculite). For this sample, both the TD and PGSE-NMR results on water diffusion were obtained to assess the concordance of the two methods on the analysis of water diffusion despite the significant difference in the timescales probed for water dynamics (i.e., ms and days for the PGSE-NMR and TD experiments, respectively). Following a coupled experimentalcomputational approach (Dabat et al., 2020), all these data were systematically compared to the results obtained by BD simulations, which were performed with 3D virtual porous media, generated to mimic the real ones in term of mineralogy and porosity distribution, while also covering a large range of anisotropy degrees in particle orientations.

\section{Materials and Methods}

\subsection{Materials}

The vermiculite used in this study is from Santa Olalla (Huelva, Spain; Gonzáles García and García Ramos, 1960; De La Calle et al., 1977). In its original form, the centimetric vermiculite crystals are $\mathrm{Mg}^{2+}$-saturated with a structural formula (Marcos et al., 2003; Arguelles et al., 2010): [( $\left.\left.\left.\mathrm{Mg}_{4.92} \mathrm{Fe}_{0.44} \mathrm{Al}_{0.60} \mathrm{Ti}_{0.04}\right)\left(\mathrm{Si}_{5.66} \mathrm{Al}_{2.34}\right) \mathrm{O}_{20}(\mathrm{OH})_{4}\right]\left(\mathrm{Mg}_{0.76} \mathrm{Ca}_{0.06} \mathrm{Na}_{0.04}\right),{ }^{2} .63 \mathrm{H}_{2} \mathrm{O}\right]$. The 0.1-0.2 $\mu \mathrm{m}$ particle size fraction, used for this study, was obtained by sonication and centrifugation methods using the protocol established by Reinholdt et al., (2013). The kaolinite used is KGa-2 from the Source Clay Repository of the Clay Mineral Society, with an average size of the particles of approximately $0.5 \mu \mathrm{m}$ (Hassan et al., 2005), and a structural formula (Mermut and Cano, 2001) of $\left[\left(\mathrm{Al}_{3.80} \mathrm{Ti}_{0.13} \mathrm{Fe}^{3+}{ }_{0.07}\right)\left(\mathrm{Si}_{3.84} \mathrm{Al}_{0.16}\right) \mathrm{O}_{5}\left(\mathrm{OH}_{4}\right)\right]$.

Both materials were exchanged into homoionic $\mathrm{Na}^{+}$form (i.e., Na-kaolinite and $\mathrm{Na}$ vermiculite) using five saturation cycles with a $1-\mathrm{M} \mathrm{NaCl}$ solution, followed by removal of excess of salt by dialysis using a 6-8 KD membrane until test with silver nitrate was negative. 
Compacted samples were prepared from the dialyzed clay dispersion dried at $60^{\circ} \mathrm{C}$ and then sieved through a $50 \mu \mathrm{m}$ mesh to remove the coarsest aggregates. A centrifuged sample made of Na-vermiculite particles was prepared directly from the dialyzed dispersion and was then stored at $4^{\circ} \mathrm{C}$.

\subsection{Preparation of samples with contrasted anisotropy in particle orientation}

The methodology used to prepare compacted Na-kaolinite for PGSE-NMR experiments as well as experimental setup and acquisition conditions are detailed in section S1.1 in Supplementary Data (S. D.). For TD experiments, the isotropic samples were prepared by uniaxial compaction of dried clay powder in (poly)methyl methacrylate (PMMA) tubes (diameter of $0.95 \mathrm{~cm}$ and a height of $7 \mathrm{~cm}$ ) as in Tertre et al. (2018). By contrast, a new protocol was developed in the present study to build an anisotropic Na-vermiculite medium with the same total porosity $(\varepsilon)$ as the one obtained by compaction. This protocol is illustrated in Fig. 1 and was developed based on the centrifugation process used by Dabat et al., (2020) to prepare Na-kaolinite samples. It first consists of transferring $3 \mathrm{~mL}$ of Na-vermiculite dispersion (20 g/L) into PMMA tube designed to be mounted directly into the TD cell to avoid sample transfer and the associated potential damage (Fig. 1a). After a horizontal centrifugation run at 23850 g for 25 minutes (Centrifuge Avanti J 301, rotor JS-24.38 from Beckman Coulter ${ }^{\circledR}$ (Fig. 1b)), the clear supernatant was removed, and a new aliquot was added (Fig. 1c). These steps were repeated ten times to obtain a sufficient mass in the tube (Fig. 1d). After centrifugation, it was necessary to dry the sample to determine its dried mass used in $\varepsilon$ calculation (details in the next paragraph). However, because drying by heating can introduce an important shrinkage of samples and distortion of their structure (Loeber, 1992; Durrieu et al., 1997), a methodology previously developed for clay cakes (Loeber, 1992; Pret, 2003) was applied to preserve, as best as possible, the geometry and particle organization in the sample. Accordingly, the sample was first flash frozen by immersion in slush nitrogen $\left(\mathrm{SN}_{2}\right)$ for $2-5$ minutes (Fig. 1e) and then dried 
by lyophilization at $-40^{\circ} \mathrm{C}$ for $2-3$ hours. Complete water removal was insured when the sample maintained a stable mass during the last hour of lyophilization (Fig. 1f). Finally, the sample was mounted to the TD cell, uniaxially compressed to the desired height (Fig. 1g-h).

The final thickness $(L)$ of all samples prepared for this study are reported in Table 1. Total porosity $\varepsilon$ was calculated as difference between the sample volume (considering the diameter of the tube and the sample's $L$ ), and the volume occupied by the solid only (i.e., clay particles), which was determined based on the sample dry mass (at the end of the lyophilization process) and clay grain density. The latter was calculated to be $2.62 \mathrm{~g} \mathrm{~cm}^{-3}$ for Na-kaolinite based on the structural formula and the crystal structure parameters refined for KGa-2 kaolinite by Sakharov et al. (2016) and $2.73 \mathrm{~g} \mathrm{~cm}^{-3}$ for Na-vermiculite using the $(a, b)$ cell parameters and the chemical composition of the 2:1 layer reported by Arguelles et al. (2010) and the $\mathrm{c}^{*}$ parameter (i.e., the basal spacing) value for dehydrated layers at $10 \AA ̊$ (Gieseking, 1975). However, Na-vermiculite displays a mono-hydrated state $(1 \mathrm{~W})$ at room humidity and a bi-hydrated state $(2 \mathrm{~W})$ in watersaturated conditions, respectively (Faurel, 2012). Consequently, two hydrated densities of 2.49 and $2.24 \mathrm{~g} \mathrm{~cm}^{3}$ for $1 \mathrm{~W}$ and $2 \mathrm{~W}$ Na-vermiculite, respectively were calculated based on water content and $c^{*}$ parameters derived by Faurel. (2012) for $1 \mathrm{~W}\left(4.13 \mathrm{H}_{2} \mathrm{O} / \mathrm{O}_{20}(\mathrm{OH})_{4}\right.$, $\left.\mathrm{c}^{*}=11.97 \AA\right)$ and $2 \mathrm{~W}$ layers $\left(9.9 \mathrm{H}_{2} \mathrm{O} / \mathrm{O}_{20}(\mathrm{OH})_{4}, \mathrm{c}^{*}=14.86 \AA\right)$. Note that for Na-kaolinite, only the interparticle porosity ( $\varepsilon_{\text {interp. }}$ ) is accessible by the water tracer, resulting in $\varepsilon=\varepsilon$ interp., whereas Na-vermiculite exhibits both $\varepsilon_{\text {interp }}$ and interlayer porosity ( $\left.\varepsilon_{\text {interl. }}\right)$. By considering the difference in the $\mathrm{c}^{*}$ parameter value between dehydrated layers at $10 \AA$ (Gieseking, 1975) and fully water saturated layers at $14.86 \AA$ (Faurel, 2012), the interlayer volume corresponds approximately to $1 / 3$ of the particle thickness in water-saturated conditions. Consequently, the proportion of the $\varepsilon_{\text {interp. }} v s$. $\varepsilon_{\text {interl. }}$ porosities for Na-vermiculite (Table 1) can be calculated for such conditions by using the following relation:

$$
\varepsilon=\varepsilon_{\text {interp. }}+\varepsilon_{\text {interl. }}=\varepsilon_{\text {interp. }}+\left(1-\varepsilon_{\text {interp. }}\right) / 3
$$




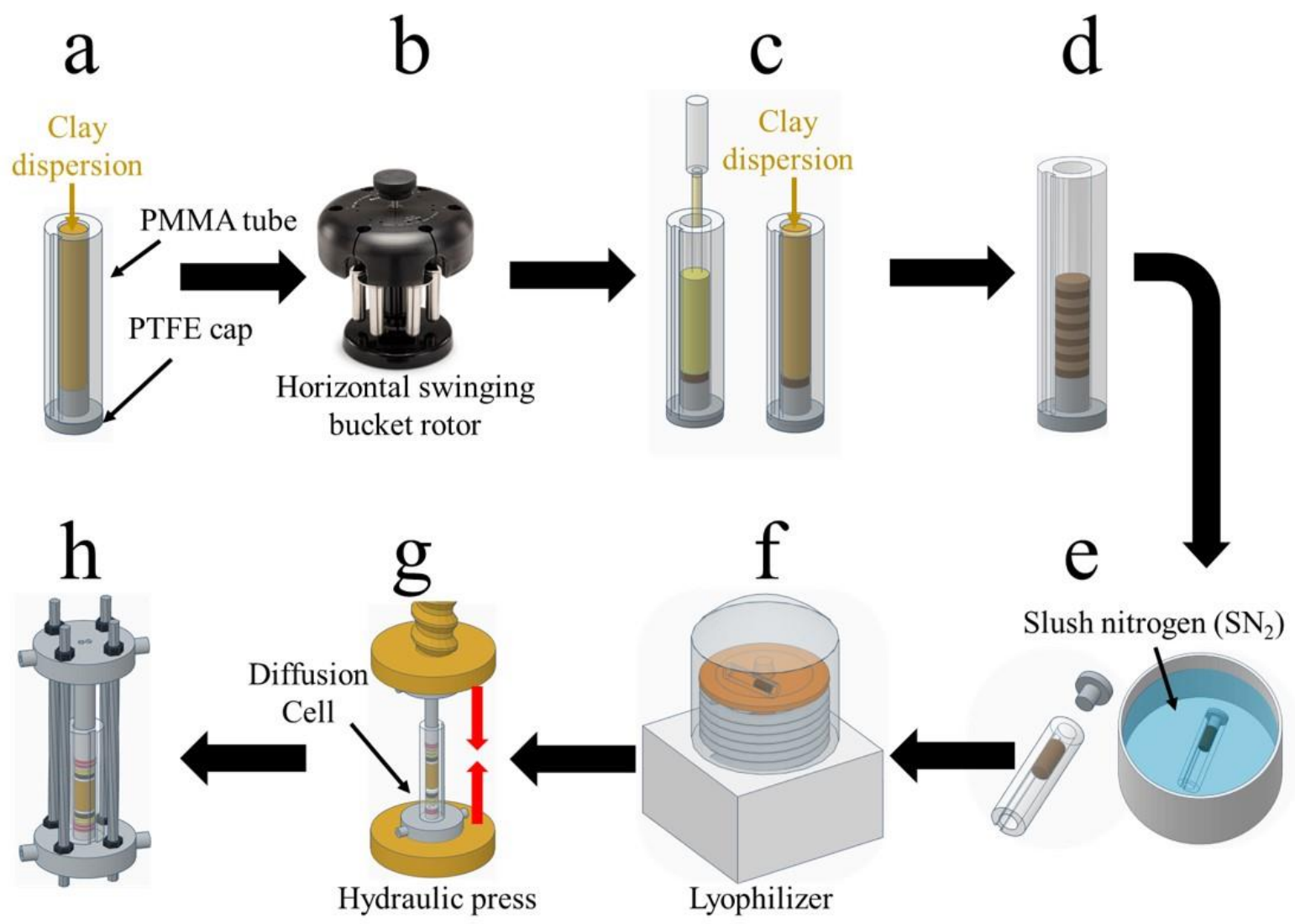

Fig. 1. Illustration of the methodology used to prepare Na-vermiculite samples with a high degree of particles' preferred orientation. (a-d) Oriented sample is prepared by 10 runs of centrifugation of Navermiculite dispersion in (poly)methyl methacrylate (PMMA) tubes sealed from the bottom with (poly)tetrafluoroethylene (PTFE) caps and by removal of the supernatant after each centrifugation run. (e-f) Preparation of slush nitrogen and freezing of the sample followed by lyophilization. (g-h) Mounting the sample into the diffusion cell and compaction to a certain thickness to reach the desired total porosity value (see Section 2.2), the final thickness of the diffusion cell is maintained by threaded rods and nuts.

\subsection{Measurements of preferred orientation of clay particles through the $\left\langle P_{2}\right\rangle$ order} parameter

The degree of anisotropy in the orientation of clay particles in the samples used in this work was obtained from two-dimensional X-Ray Scattering (2D-XRS) patterns recorded from the duplicate samples. The measurements were performed at the Laboratoire de Physique des Solides (LPS) - Orsay, France. Sample preparation method (i.e., induration and slicing) and 
2D-XRS acqu isition parameters are detailed in S. D. (S.1.2). In this work, the degree of anisotropy in the orientation of clay platelets is reported in terms of $\left\langle\mathrm{P}_{2}\right\rangle$ order parameter values also referred to as the nematic order $S$ (Dabat et al., 2018; Underwood and Bourg, 2020) or the Hermans parameter $H$ (Hermans and Platzek, 1939). The $\left\langle\mathrm{P}_{2}\right\rangle$ order parameter can be derived from the orientation distribution functions (ODF) (Dabat et al., 2019, 2020 and references therein) obtained from 2D-XRS patterns. Briefly, in a spherical coordinate, the orientation of a platelet shape clay particle can be defined as a function of two angles: $\theta$, which is the azimuthal angle between the normal of the particle (vector that is perpendicular to the basal surface of the particle) and the $\mathrm{z}$ axis, and $\Phi$, the polar angle in the (xy) plane (Fig. 2a). For particles with uniaxial symmetry (i.e., clay particles for which their $\mathrm{z}$ axis is the only symmetry axis as in this study, Wenk et al. (2010) and references therein) the probability for a particle orientation over all possible $\Phi$ angle values is equal. Therefore, the orientation of a clay platelet (ODF) can be described as a function of only its $\theta$ angle, i.e., $f(\theta)$, with the following constraints:

$$
\begin{gathered}
f(\theta) \geq 0 \\
f(\theta)=f(\pi-\theta) \\
\int_{0}^{\pi} f(\theta) \sin (\theta) d \theta=1
\end{gathered}
$$

Eqs. (2) and (3) indicate that the ODF is always positive, with the same probability of the particles pointing upward or downward. The term $f(\theta) \sin (\theta)$ in Eq. (4) corresponds to the normalization over all the particles in the sample. From the 2D-XRS patterns, the scattered intensities ( $I$ ) of the 001 Bragg peak modulated as a function of the angle $\tau$ (the angle between the normal of the incident beam and the surface of the 2D-detector), and the angle $\theta$ (Fig. 2b). Because of the position of the 001 reflection at low scattering angles $\left(2 \theta_{\mathrm{B}}\right)$, the hypothesis $\theta=$ $\tau$ is a valid approximation (Dabat et al., 2019). Hence, the ODF $f(\theta)$ can be expressed from the scattered intensity $I(\theta)$ evolution integrated from the 2D-XRS patterns as follows: 
261 The ODF $f(\theta)$ can also be written with a second order Legendre polynomial as follows:

$$
\left\langle P_{2}\right\rangle=\int_{0}^{\pi}\left\langle P_{2}\right\rangle(\cos \theta) \cdot f(\theta) \sin (\theta) d \theta
$$
organization with all particles having the same preferred orientation (i.e., bedding plane).

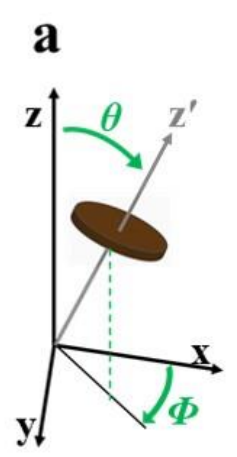

\section{c}

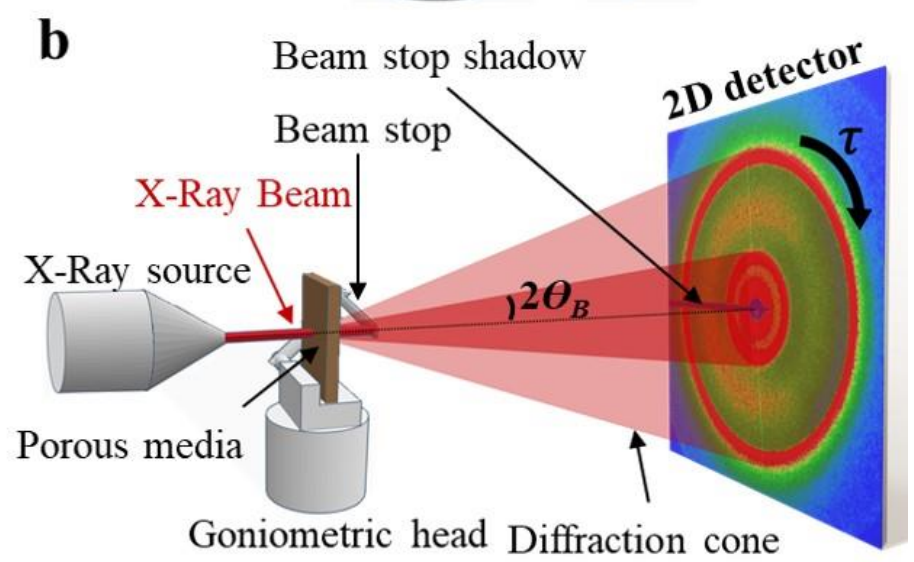

Fig.2.

Experimental measurement of the particle orientation in clayey samples using an X-ray scattering technique with a 2D-detector (2D-XRS). (a) Spherical coordinates ( $\rho$, $\theta, \Phi)$ with respect to the sample tube (laboratory frame: $(\mathrm{x}, \mathrm{y}, \mathrm{z}))$ and the angles $(\theta, \Phi)$ describing the orientation of the normal ( $\mathrm{Oz}$ ') of an individual clay particle. (b) The 2D-XRS instrumental setup and measurement of the particle orientation by azimuthal integration (along $\tau$ ) of the scattered intensity. (c) Illustration of the method to cut the indurated sample into lamellas: (i) the indurated sample is cut into two vertical halves, (ii) cutting the longitudinal and transversal lamellas (the cutting planes are shown in light colour cuboid). The lamellas are reduced to a thickness of $500 \mu \mathrm{m}$ by polishing.

\subsection{Water diffusion measurement in Na-vermiculite and kaolinite samples}

Experimental water diffusion coefficients were obtained from $\mathrm{Na}$-vermiculite and $\mathrm{Na}$ kaolinite samples using PGSE-NMR and TD of the water tracer (HDO) experiments. Sample preparation, acquisition parameters, and data treatment are detailed in the S. D. (sections S.1.1 
the effective diffusion coefficient $D e$ of HDO in the $z$-direction of the tube $\left(D e_{z}\right)$, i.e., perpendicular to the compression direction/centrifugation plane of the sample, while the PGSENMR analysis allows to obtain the pore diffusion coefficients $D p_{z}$ and $D p_{x, y}$.

$D e_{z}$ is defined from the self- diffusion coefficient of species in bulk water $\left(D_{0}\right)$ by the following equation (Bear, 1972; Dykhuizen and Casey, 1989; Bourg et al., 2006):

$$
D e=\frac{\varepsilon}{G} D_{0}
$$

where $\varepsilon$ is the accessible porosity for the tracer, and $G$ the geometrical factor (tortuosity factor), which describes the impact of the pore-network geometry (pore shape, particle orientation, connectivity, and constrictively). The pore diffusion $D p$ can also be related to $D_{0}$ as follows:

$$
D p=\frac{1}{G} D_{0}
$$

Hence, in the case of water tracers for which the total porosity is accessible to diffusion and no adsorption occurs, the pore and effective diffusion coefficients are related to $\varepsilon$ as follows:

$$
D e=\varepsilon D p
$$

\subsection{Brownian dynamics simulations of water diffusion in virtual porous media}

Mesoscale Brownian dynamics simulations of water diffusion in both Na-vermiculite and Na-kaolinite as a function of the anisotropy in the particles' orientation were performed on 3D virtual porous media (VPM). To cover a large range of anisotropy degrees in particle orientations, 13 particle packings were generated with $\left\langle\mathrm{P}_{2}\right\rangle$ values varied from 0.03 to 0.96 (Ferrage et al., 2018; Dabat et al., 2020); see Table. S1 in S. D., and Fig. 3a), mimicking the distribution of the shapes and sizes of the particles in these samples. The description of the generation of these VPM is detailed in S.1.4 of S. D.

Brownian dynamics simulations were performed according to the methodology detailed by Dabat et al. (2020). For the case where the time step $\delta t$ is much larger than the velocity 
correlation time $\gamma_{i}$ of probe $i$ (i.e., $\gamma_{i} \delta t \gg 1$ ), and considering the probe's displacements as completely independent, the displacement of molecular probes in the framework of the general Langevin equation can be reduced as follows (Gunsteren et al., 1981):

$$
\vec{x}_{i}(t+\delta t)=\vec{x}_{i}(t)+\vec{R}_{i}
$$

with the random force $\vec{R}_{i}$ that satisfies a Gaussian distribution function with zero mean and standard deviation given by

$$
\left\langle R_{i}^{2}\right\rangle=2 D_{i} \delta t
$$

where $D_{i}$ is the molecular self-diffusion of probe $i$. For diffusion within unrestricted isotropic media, the modulus of the random $3 \mathrm{D}$ displacements is quantified by the self-diffusion propagator, i.e., the density of the probability of the displacement distribution law (Callaghan, 1991; Bacle et al., 2016):

$$
P\left(\vec{r}, \Delta \mid \vec{r}_{0}, 0\right)=\{4 \pi D \Delta\}^{-3 / 2} \exp \left[-\frac{\left(\vec{r}-\vec{r}_{0}\right)^{2}}{4 D \Delta}\right]
$$

where $\Delta$ is the diffusion time. The self-diffusion propagator that quantifies the mobility along a single director is then defined by

$$
P_{\alpha}\left(\overrightarrow{r_{\alpha}}, \Delta \mid \overrightarrow{r_{\alpha}}, 0\right)=\left\{4 \pi D_{\alpha} \Delta\right\}^{-1 / 2} \exp \left[-\frac{\left(\overrightarrow{r_{\alpha}}-{\overrightarrow{r_{\alpha}}}_{0}\right)^{2}}{4 D_{\alpha} \Delta}\right]
$$

where $D_{\alpha}$ is the component of the self-diffusion tensor that describes the mobility of the probe along the selected $\overrightarrow{e_{\alpha}}$ director (Callaghan, 1991).

For the BD simulations of water diffusion in Na-kaolinite with only interparticle porosity, the same methodology as used by Dabat et al. (2020) was applied. However, in contrast to simulations performed in this latter study, the size of the simulation box was not adapted to the size of the kaolinite particles. Indeed, in the absence of grain porosity and without consideration of specific interfacial effects on the probe dynamics, a mesoscopic simulation is independent of the particle size, given that the time step is correctly adapted to the modification of the length scale. Accordingly, the same simulation box as for Na-vermiculite (Table S1) was used to 
compute the water diffusion in the kaolinite samples. Such a consideration is also supported by the similar aspect ratios of particles for both types of minerals ( 0.08 and 0.07 for $\mathrm{Na}$-vermiculite and kaolinite, respectively) (Hassan et al., 2005; Reinholdt et al., 2013). For interparticle diffusion in a kaolinite sample, a number $N=2000$ of water probes with molecular selfdiffusion $D_{0}$ set at $2.3 .10^{-9} \mathrm{~m}^{2} \mathrm{~s}^{-1}$ were introduced randomly into the interparticle porosity of the VPMs. A time step $\delta t=200 \mathrm{ps}$, over a total simulation time of $0.12 \mathrm{~ms}$, was considered, and additional tests showed that smaller values did not provide a difference in the simulation results. During the simulations, the collision with the surface of the Na-kaolinite particles was treated as a Maxwell's $A$ wall with the angular probability $f_{A}(\theta, f)$ of water probes rebounding from the surface given by

$$
f_{A}(\theta, \phi) d \Omega=\cos \theta d \Omega / \pi
$$

where $\theta$ and $f$ are the usual polar and azimuthal angles (with respect to the normal of the surface of the particle), and $d \Omega$ is the element of the solid angle (Fig. 3b). That condition mimics the trajectory of a molecule by assuming a residence time at the solid surface that is long enough to thermalize its velocity distribution function before being desorbed and returning to the fluid (Valleau et al., 1991).

For BD simulations of water diffusion in Na-vermiculite, the water probes were allowed

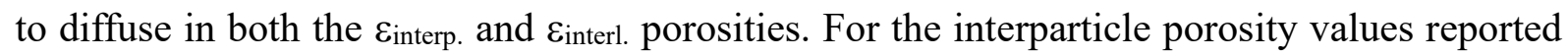
in Table S1, the total porosity of the VPM is calculated according to Eq. (1). The self-diffusion coefficients for the water molecules in the interlayer space $\mathrm{D}_{\text {interl. }}$ were extracted from previous molecular dynamics simulations performed by Tertre et al. (2015) for Na-vermiculite. Although these authors only reported the self-diffusion coefficients for $\mathrm{Na}^{+}$, the associated bi-dimensional self-diffusion coefficient for the water molecules was found to be at $D_{\text {interl. }}=2.3 \cdot 10^{-10} \mathrm{~m}^{2} \mathrm{~s}^{-1}$. Such a value is one order of magnitude lower than that of bulk water and is in agreement with numerical MD results and experimental quasi-elastic neutron scattering results obtained for 
tetrahedrally-charged trioctahedral saponite with different layer charges (Michot et al., 2012).

Note that the obtained $D_{\text {interl. }}$ value is lower than commonly reported for montmorillonite (Swenson et al., 2000; Marry and Turq, 2003; Malikova et al., 2005, 2006; Skipper et al., 2006), related to the lower charge and location of the charge in the octahedral sheet of this mineral. For the initial configuration of the BD simulations, the 2000 water probes were randomly introduced in the simulation box with an acceptance probability of $1 / 3$ when the probe was included in the solid. The same time step and simulation time as for water diffusion restricted to the interparticle porosity only was considered. During the simulation (Fig. 3c), the probes colliding with the basal surface of the particles were allowed to rebound according to Eq. (11), whereas a specific condition occurred in the case where the probe was in contact with a lateral surface. In such a condition, based on a system at equilibrium (Bacle et al., 2016), the probe was allowed to penetrate (or leave) the interlayer space of the particle according to the acceptance rule:

$$
p_{a c c}^{i \rightarrow j}=\min \left[1 ; \frac{p^{j}}{p^{i}} \times \frac{p_{g e n}^{j \rightarrow i}}{p_{g e n}^{i \rightarrow j}}\right]=\min \left[1 ; \frac{c_{j}}{c_{i}} \times\left(\frac{D_{i}}{D_{j}}\right)^{3 / 2} \times \exp \left(-\frac{\ell^{2}}{4 D_{j} \delta t}+\frac{\ell^{2}}{4 D_{i} \delta t}\right)\right]
$$

where $\ell$ is the modulus of the displacement from Eq. (11), and $\mathrm{c}_{\mathrm{i}}$ and $\mathrm{c}_{\mathrm{j}}$ are the water probe concentrations in the environments $i$ and $j$, respectively, with self-diffusion coefficients $D_{i}$ and $D_{j}$. In the case of the probe that did not satisfy the acceptance rule to penetrate in the interlayer porosity, a rebound was then considered according to Eq. (14) (Fig. 3c).

For simulations of water diffusion in both Na-kaolinite and Na-vermiculite, the pore water diffusion coefficients $D p_{\alpha}$ along a selected $\overrightarrow{e_{\alpha}}$ director were extracted from the asymptotic slope of the radial mean squared displacement, as follows:

$$
D p_{\alpha}=\lim _{\Delta \rightarrow \infty} \frac{\sum_{i=1}^{N}\left(x_{i, \alpha}(0)-x_{i, \alpha}(\Delta)\right)^{2}}{2 \mathrm{~N} \Delta}
$$

where $\alpha$ refers to the single direction $\overrightarrow{e_{\alpha}}$ on which the mobility is extracted (other parameters were already defined above; uncertainty on the calculated $D p_{\alpha}$ values were estimated at $10 \%$ ). 

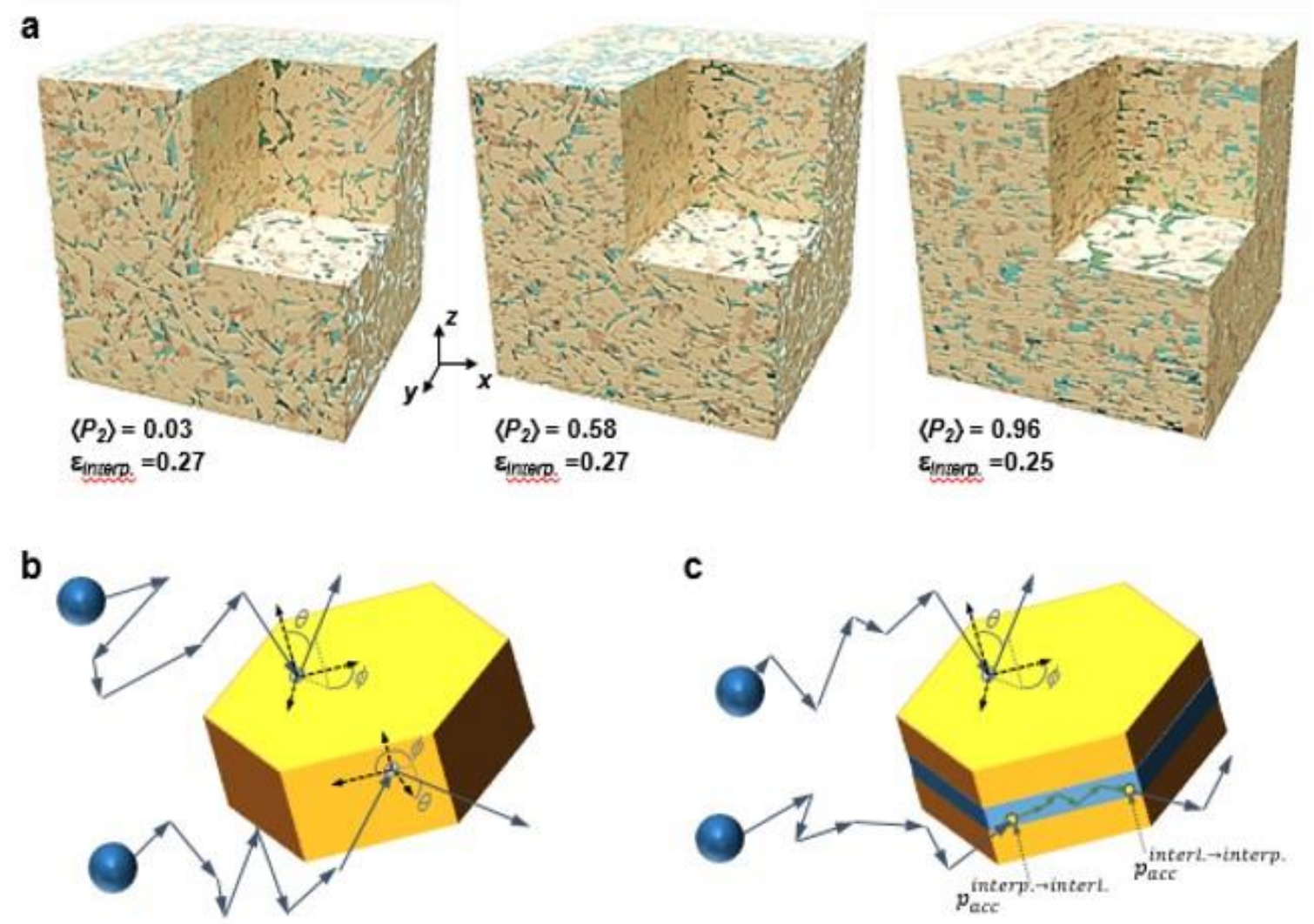

Fig. 3. (a) Virtual porous media with similar interparticle porosity ( $\left.\varepsilon_{\text {interp. }}\right)$ values but different anisotropy degrees in particle orientation (i.e., $\left\langle\mathrm{P}_{2}\right\rangle$ order parameter). (b) Illustration of Brownian dynamics displacements of water molecules in an Na-kaolinite sample. Water probes, with a self-diffusion coefficient $\mathrm{D}_{0}$, diffuse in the interparticle porosity. A rebound of the water probe on a basal or lateral surface of the particle is treated according to Maxwell's A wall (see text for details). (c) Brownian dynamics of water diffusion in Na-vermiculite. The water probe, initially in the interparticle porosity and diffusing with $\mathrm{D}_{0}$ as the self-diffusion coefficient, can either rebound on the basal surface or penetrate the interlayer space of the particle according to the acceptance rule $p_{\text {acc }}^{\text {interp. } \rightarrow \text { interl. }}$. If the acceptance rule is satisfied, the probe then diffuses in the interlayer space according to the self-diffusion coefficient $\mathrm{D}_{\text {interl. }}$, which was extracted from molecular dynamics. Eventually, the water probe can leave the particle and go back to the interparticle porosity according to the acceptance rule $p_{\text {acc }}^{\text {interl } \rightarrow \text { interp. }}$.

\section{Results and discussion}

\subsection{Measurement of preferred orientation of clay particles}

For each sample, longitudinal and transverse 2D-XRS patterns were recorded (Fig. 2b, c) with respect to the sample tube axis (i.e., the direction of compaction/preferred orientation of clay particles) (Fig. 2a). These patterns are reported in Fig. 4a with their corresponding ODFs 
samples, the transverse ODFs (in gray) remain approximately constant regardless of the $\theta$ angle

(Fig. 4b), which indicates that the transverse lamellas are isotropic, as expected for lamellas anisotropy in the particles' orientation in this direction.

Table 1. Experimental results for water tracer (HDO) in Na-kaolinite and Na-vermiculite from through414 Diffusion (TD) and ${ }^{1} \mathrm{H}$ NMR pulsed gradient spin echo (PGSE-NMR) experiments, and the properties of the porous media used (material, thickness, porosity, degree in preferred orientation).

\begin{tabular}{lcccc}
\hline & Na-kaolinite & Na-kaolinite & Na-vermiculite & Na-vermiculite \\
\hline Preparation method & Compaction & Compaction & Compaction & Centrifugation \\
Experimental technique & PGSE-NMR & TD & TD & TD \\
Sample thick., $L(\mathrm{~mm})$ & $10.00 \pm 0.10$ & $6.00 \pm 0.10$ & $7.99 \pm 0.10$ & $6.44 \pm 0.10$ \\
Total porosity, $\varepsilon$ & $0.26 \pm 0.02$ & $0.26 \pm 0.02$ & $0.49 \pm 0.02$ & $0.49 \pm 0.02$ \\
Interparticle poro., $\varepsilon_{\text {interp. }}$ & $0.26 \pm 0.02$ & $0.26 \pm 0.02$ & $0.24 \pm 0.02$ & $0.24 \pm 0.02$ \\
Interlayer poro., $\varepsilon_{\text {interl. }}$ & $0.00 \pm 0.02$ & $0.00 \pm 0.02$ & $0.26 \pm 0.02$ & $0.26 \pm 0.02$ \\
Order parameter, $\left\langle\mathrm{P}_{2}\right\rangle$ & $0.34 \pm 0.02$ & $0.34 \pm 0.02$ & $0.03 \pm 0.02$ & $0.41 \pm 0.06$ \\
$D p_{z}{ }^{\mathrm{a}}\left(\times 10^{-10} \mathrm{~m}^{2} \mathrm{~s}^{-1}\right)$ & $5.77(5.19-6.35)$ & $6.92(5.38-9.23)$ & $3.61(3.06-4.49)$ & $1.82(1.37-2.27)$ \\
$D e_{z}{ }^{\mathrm{b}}\left(\times 10^{-10} \mathrm{~m}^{2} \mathrm{~s}^{-1}\right)$ & $1.50(1.35-1.65)$ & $1.80(1.40-2.40)$ & $1.80(1.50-2.20)$ & $0.89(0.67-1.11)$ \\
$D p_{z} / D_{0}{ }^{\mathrm{c}}$ & $0.29(0.26-0.32)$ & $0.34(0.26-0.45)$ & $0.18(0.15-0.22)$ & $0.09(0.07-0.11)$ \\
$D e_{z} / D_{0}{ }^{\mathrm{d}}$ & $0.08(0.07-0.08)$ & $0.09(0.07-0.12)$ & $0.09(0.07-0.11)$ & $0.04(0.03-0.05)$ \\
$\alpha^{\mathrm{e}}$ & - & $0.30(0.25-0.34)$ & $0.49(0.40-0.80)$ & $0.49(0.40-0.70)$ \\
$K_{\mathrm{d}}{ }^{\mathrm{f}}\left(\mathrm{mL} \mathrm{\textrm {g } ^ { - 1 } )}\right.$ & - & $0.02(0.00-0.04)$ & $0.00(0.00-0.22)$ & $0.00(0.00-0.15)$ \\
\hline
\end{tabular}

${ }^{a}$ Pore diffusion coefficient of water in the direction perpendicular to the preferred orientation of the particles.

${ }^{\mathrm{b}}$ Effective diffusion coefficient of water in the direction perpendicular to the preferred orientation of the particles.

${ }^{\mathrm{c}}$ Pore diffusion coefficient of water normalized to the self-diffusion coefficient of HDO in bulk water. ${ }^{\mathrm{d}}$ Effective diffusion coefficient of water normalized to the self-diffusion coefficient of HDO in bulk water.

${ }^{\mathrm{e}}$ Rock capacity factor (see Eq. (S2) in S.D.).

${ }^{\mathrm{f}}$ Distribution coefficient (see Eq. (S2) in S.D.). 
particles in this sample. The corresponding $\left\langle\mathrm{P}_{2}\right\rangle$ value of $0.34 \pm 0.025$ was averaged from several measurement points. For Na-vermiculite samples, the corresponding longitudinal ODFs

420 display different shapes, as reflected by their contrasted $\left\langle\mathrm{P}_{2}\right\rangle$ values of $0.03 \pm 0.025$ and $0.41 \pm 0.06$ for the compacted and centrifuged samples, respectively. For the purpose of this study, the contrast in the $\left\langle\mathrm{P}_{2}\right\rangle$ values between the two samples are sufficient for investigating the effect of the particles' organization on the diffusion of HDO. Uncertainties on the $\left\langle\mathrm{P}_{2}\right\rangle$ values were considered to be the standard deviation (STD) of a population of several measurement points from the same sample.

The relatively higher uncertainty for the $\left\langle\mathrm{P}_{2}\right\rangle$ values obtained from the centrifuged sample with respect to the compacted values ( \pm 0.06 and \pm 0.025 , respectively) can be attributed to the influence of the preparation method (uniaxial compaction $v s$ centrifuging). For example, in the case of a centrifuged sample, with a slight variation in the solid-solution ratio, hydrostatic or shear forces, and particles segregation between successive centrifuging cycles led to formation of successive beds with some heterogeneity in the particles' preferred orientation among them. Similarly, Dabat et al. (2020) also reported a slightly higher uncertainty for the $\left\langle\mathrm{P}_{2}\right\rangle$ value of centrifuged Na-kaolinite samples (i.e., anisotropic) than for the more isotropic values obtained by compaction.

\subsection{Water diffusion in Na-kaolinite}

The HDO mobility in Na-Kaolinite samples $\left(\varepsilon=0.26 \pm 0.025,\left\langle\mathrm{P}_{2}\right\rangle=0.34 \pm 0.02\right)$ was investigated in the direction of the compaction axis (normal to the preferred orientation of clay particles) with two analytical methods: PGSE-NMR and TD (see sections 2.4). The results from both experiments are reported in terms of the pore diffusion coefficient $\left(D p_{z}\right)$ and effective diffusion coefficient $\left(D e_{z}\right)$ (definitions in Section 2.4) in Table.1. 


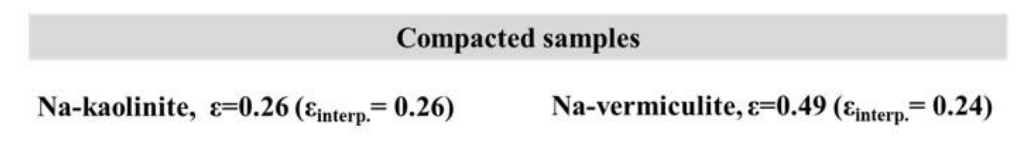

a
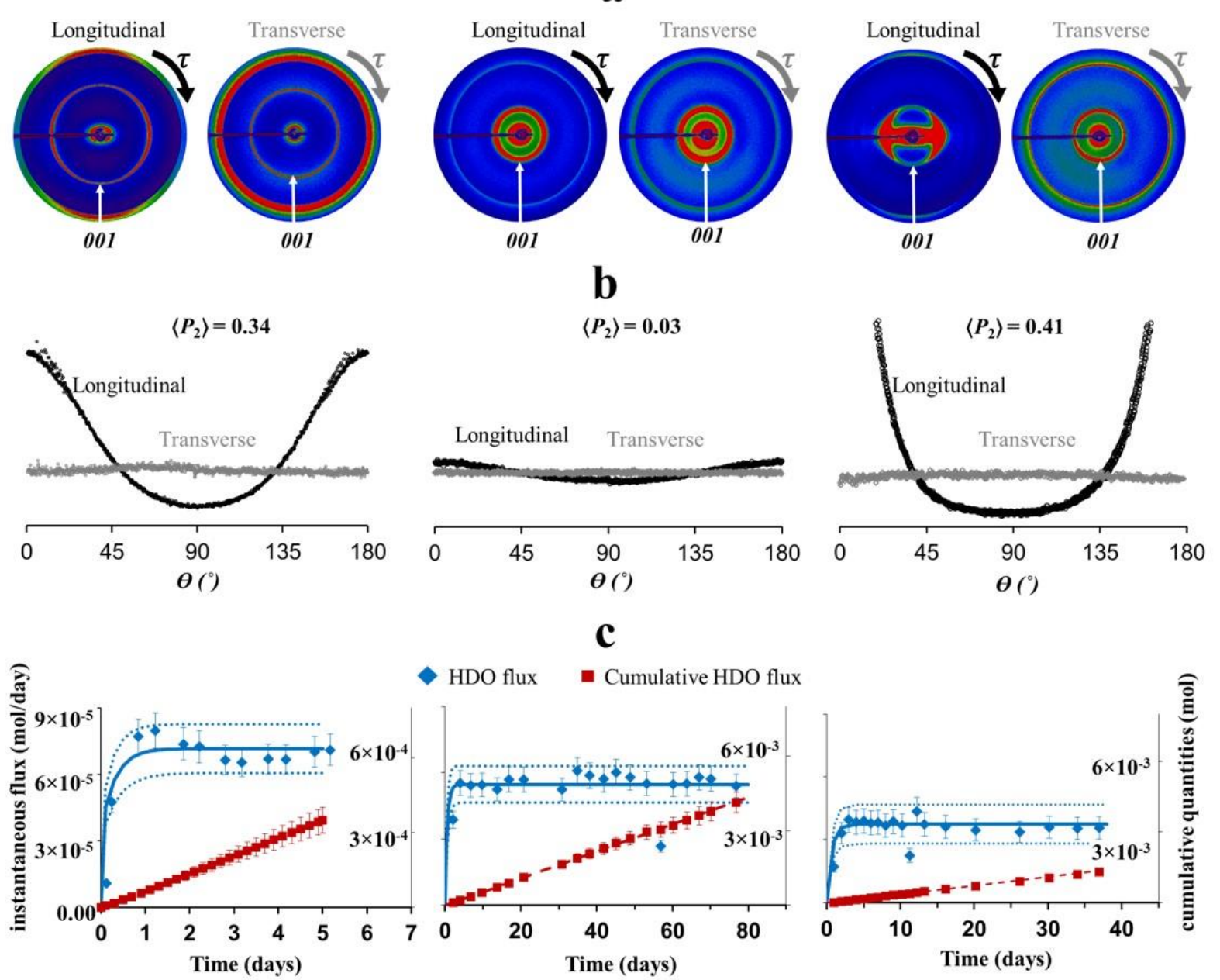

c
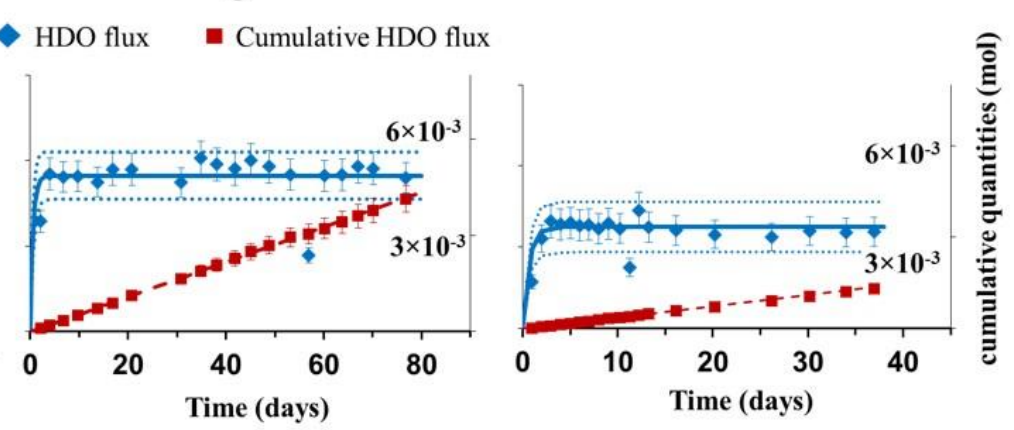

Fig. 4. Results from particle orientation and water tracer (HDO) through-diffusion (TD) measurements for Na-kaolinite and Na-vermiculite samples. (a) 2D-patterns obtained by X-ray scattering technique from longitudinal and transverse lamellas (description in Fig. 2). Color scale ranges from light gray to black with increasing scattered intensity. (b) Orientation distribution functions (ODF) extracted from an azimuthal scan over $180^{\circ}$ along the 001 reflection from longitudinal (black) and transverse (gray) lamellas. The intensities are normalized to the same value for comparison. (c) Experimental instantaneous flux (black diamonds) and cumulative quantities (gray squares) of HDO diffusing through the samples. The solid lines correspond to the fit of the experimental flux, whereas dotted lines are simulated flux curves calculated by considering the error range in the diffusion parameters (Table 1). Dashed lines correspond to simulated cumulative curves by considering the diffusion parameters interpreting the average experimental flux. The scale for the instantaneous flux axis is the same for all of the charts.

Data reported from Tertre et al. (2018) concerning the instantaneous flux of HDO measured in the downstream reservoir during TD experiments in this sample are shown in Fig. 4c. These data were interpreted with a $D e_{z}$ equal to $1.8(1.4-2.4) \times 10^{-10} \mathrm{~m}^{2} \mathrm{~s}^{-1}$ and a rock 
capacity factor $(\alpha)$ value equal to $\varepsilon(\alpha=0.30(0.25-0.34) v s . \varepsilon=0.26 \pm 0.02)$, which allows us to confirm that HDO is an inert tracer for this sample (i.e., no significant HDO adsorption).

Considering the uncertainties associated for each type of measurement $(\sim 10$ and $\sim 15 \%$ for PGSE-NMR and TD, respectively), both techniques resulted in a similar $D p_{z}$ for HDO in Na-kaolinite samples (6.9 (5.4-9.2), and $5.8(5.2-6.4) \times 10^{-10} \mathrm{~m}^{2} \mathrm{~s}^{-1}$, respectively). This similarity indicates that both techniques, even though they probe very contrasted time scales (days for TD $v s$ ms for PGSE-NMR), can be used interchangeably to investigate the dynamics of water tracer in samples composed of non-charged clayey particles irrespective of the differences in the spatial/temporal scales investigated. Accordingly, the PGSE-NMR method can be used for upscaling purposes, at least up to the centimeter scale investigated by the TD method. Moreover, similar $D p_{z}$ values mean that the two samples (the one prepared for PGSE-NMR analysis in this study and that used by Tertre et al. (2018) for TD experiments) have more or less the same porosity and degree of anisotropy in their particle orientations (considering the uncertainties on both parameters).

The $D p_{z} / D_{0}$ values issued from the TD and PGSE-NMR experiments analysis are compared to those obtained from BD simulations using VPMs $(\varepsilon=\varepsilon$ interp. $=0.25-0.27$; see Table $\mathrm{S} 1$ ), which are characterized by different $\left\langle\mathrm{P}_{2}\right\rangle$ parameter values (Figure 5a). Overall, the BD simulations show a decrease in $D p_{z}$ (measured in the direction normal to the preferred orientation) with the degree of anisotropy of the particles' orientation, in link with the increased tortuosity of the diffusion pathway. The good agreement between the experimental and simulated data obtained in this study for an interparticle porosity $\varepsilon_{\text {interp. }}$ close to 0.25 but also for a higher porosity value (i.e., 0.5; Dabat et al. (2020)) implies that the different VPMs generated and characterized by different porosity and particle organizations are well representative of the real samples, despite the difference in length scales between the real samples and VPM's (Ferrage et al., 2015). BD simulations on VPMs can thus be considered a 
reliable tool to investigate water diffusion in clayey samples, especially in situations that are experimentally challenging to produce, such as samples that are characterized by different porosities and a high degree of anisotropy in the particle preferred orientation, since, most often, the two parameters are coupled (Dabat et al., 2018).

\subsection{Water diffusion in Na-vermiculite}

TD results obtained with Na-vermiculite samples were fitted with averaged $\alpha$ values equal to $\varepsilon$, resulting in $K_{\mathrm{d}}=0$ (Table 1 ), which indicates that HDO can be considered an inert tracer in Na-vermiculite samples, as also reported for kaolinite. However, slight HDO adsorption cannot be excluded, as suggested by the higher uncertainties obtained on the $\alpha$ values for Na-vermiculite samples compared to the kaolinite values. Such behavior was also reported by Tertre et al. (2018) for Na-vermiculite samples and can be attributed to slight HDO adsorption in the interlayer of Na-vermiculite in relation to its high layer charge.

For the same $\varepsilon$ value $(\varepsilon=0.49 \pm 0.025)$, the contrast in the particles' preferred orientation (Fig. 4b) between the compacted and centrifuged Na-vermiculite samples $\left(\left\langle\mathrm{P}_{2}\right\rangle=0.03 \pm 0.02\right.$, and $0.41 \pm 0.06$, respectively) is well reflected by the difference in their experimental $D p_{z}$ values (Table 1). Indeed, the HDO flux (Fig. 4c) through the compacted sample can be interpreted with a $D p_{\mathrm{z}}$ value equal to $3.6(3.0-4.5) \times 10^{-10} \mathrm{~m}^{2} \mathrm{~s}^{-1}$, in comparison to almost two times lower $D p_{\mathrm{z}}$ at $1.8(1.4-2.3) \times 10^{-10} \mathrm{~m}^{2} \mathrm{~s}^{-1}$ obtained with the centrifuged sample. Such results indicate that the different Na-vermiculite samples were well-adapted for investigating, with similar total porosity, the influence of anisotropy in the particles' preferred orientation on the diffusion of water in a charged/dual porosity clayey media. Moreover, the data also infer that in a clayey media, and together with porosity, the preferred orientation of the particles acts as a first-order parameter in the control of the water diffusion. This behavior, previously reported by Dabat et al. (2020) for non-charged clayey samples (i.e., Na-kaolinite), therefore also extends to the case of charged clayey samples with a dual porosity. These findings decisively 
show that comprehensive data on the orientation of the particles in a clay-rich media is a prerequisite to correctly predict water diffusion within it. Finally, all the above stresses the need to develop diffusion models in which both the porosity and the degree of particle preferred orientation are explicitly included to increase the predictive capacity of such models.

In Fig. 5b and similar to Na-kaolinite samples, a good agreement is observed between experimental points from Na-vermiculite samples and simulated data $(\varepsilon=0.50-0.51$ and $\mathcal{E}_{\text {interp. }}=0.25-0.27$; Table S1). Moreover, despite the higher $\varepsilon$ value for Na-vermiculite compared to Na-kaolinite, the lower $D p_{z}$ values obtained for Na-vermiculite clearly highlight the role played by the interlayer porosity on the overall reduced mobility of the water molecules in the vicinity of charged clay particles (Nakashima and Mitsumori, 2005; Michot et al., 2012; Porion et al., 2014). Note that in Fig. 5b, the point obtained for the centrifuged Na-vermiculite sample at $\left\langle\mathrm{P}_{2}\right\rangle=0.41$ is somewhat lower than the corresponding simulated point. Such small aberration can be due to the slightly lower interparticle porosity in the case of the experimental Na-vermiculite sample $\left(\varepsilon_{\text {interp. }}=0.24\right)$ compared to that used for the BD simulation $\left(\varepsilon_{\text {interp }}=0.25-0.27\right)$. In addition, one can consider that due to the increased heterogeneity for the centrifuged sample, which is characterized by the wider variation in $\left\langle\mathrm{P}_{2}\right\rangle$ values, water diffusion could be controlled by zones in the samples with higher $\left\langle\mathrm{P}_{2}\right\rangle$ values and not by the mean degree of preferred orientation. Despite this marginal difference, the good agreement between the experimental and calculated $D p_{\mathrm{z}}$ values can be assigned to the lack of osmotic swelling in the Na-vermiculite sample, which allows a correct quantitative description of the different types of porosities volumes. In this respect, BD simulations on such granular virtual systems can be considered to be a useful tool for investigating the role played by different parameters (i.e., porosity, orientation of particles, and dual porosity volumes) on the overall diffusion process of water in Na-vermiculite dual porosity media. 
a

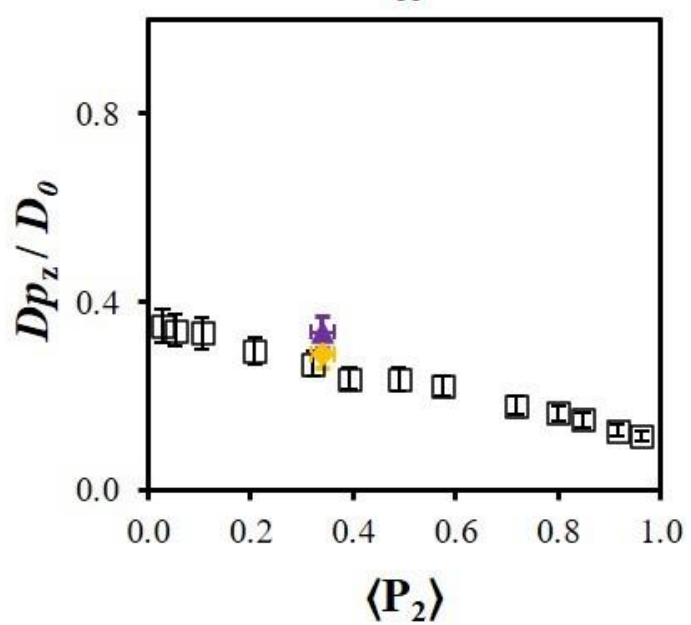

b

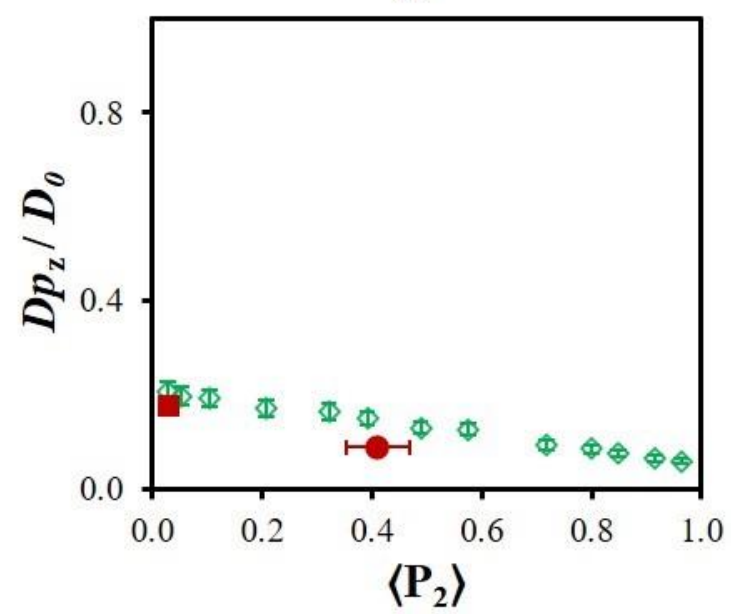

c

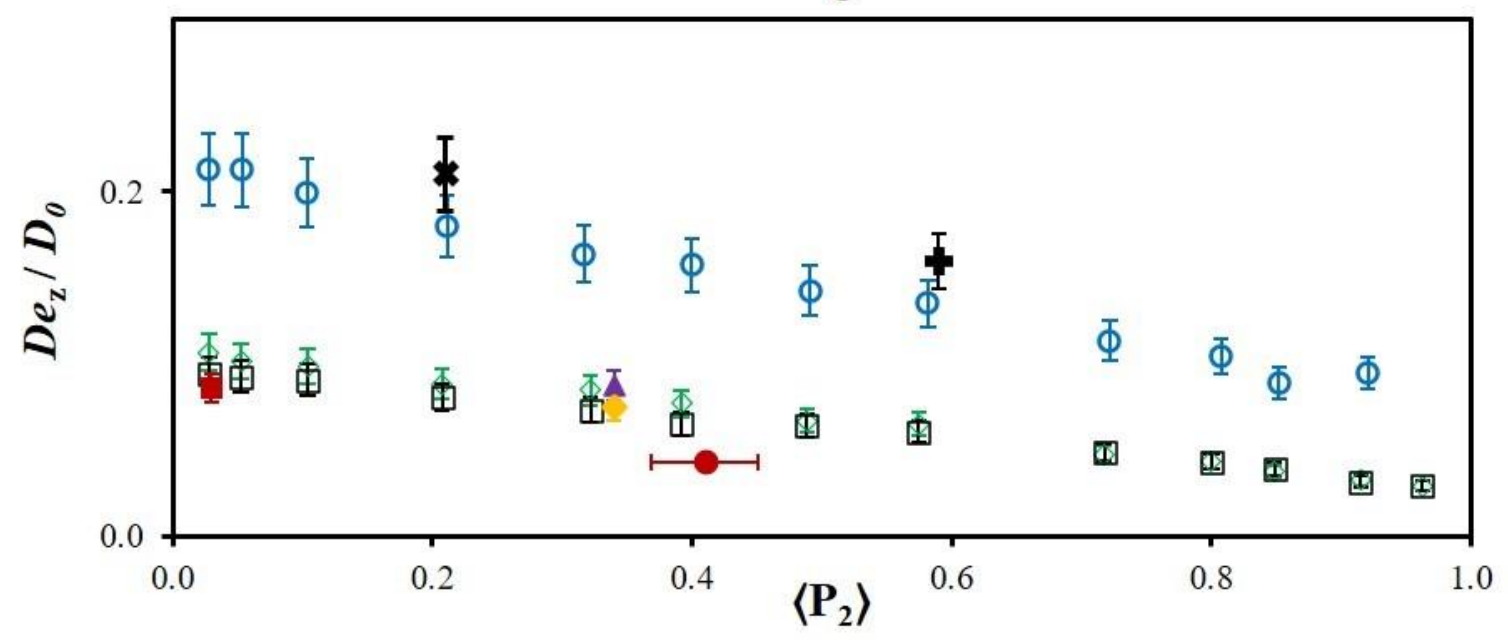

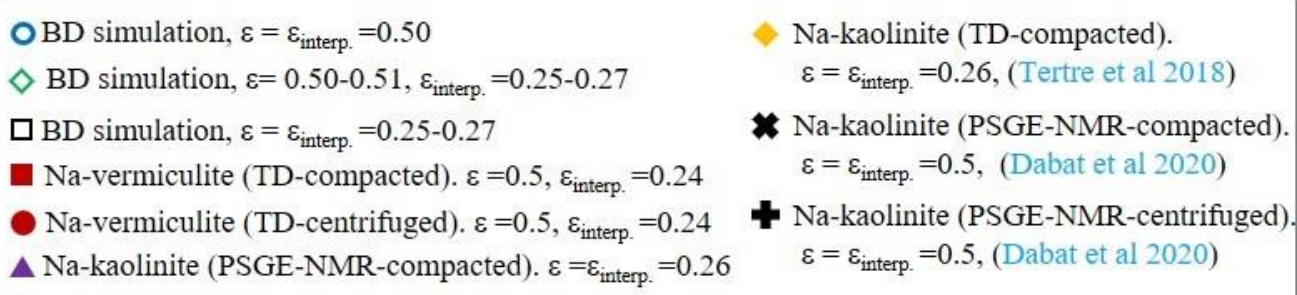

Na-kaolinite (TD-compacted). $\varepsilon=\varepsilon_{\text {interp. }}=0.26$, (Tertre et al 2018)

Na-kaolinite (PSGE-NMR-compacted). $\varepsilon=\varepsilon_{\text {interp. }}=0.5$, (Dabat et al 2020)

Na-kaolinite (PSGE-NMR-centrifuged) $\varepsilon=\varepsilon_{\text {interp. }}=0.5$, (Dabat et al 2020)

Figure 5. Comparison between experimental and Brownian dynamics (BD) simulated water diffusion coefficients in the direction perpendicular to the preferred orientation of clay particles as a function of order parameter $\left\langle\mathrm{P}_{2}\right\rangle$ values. All diffusion coefficients are normalized to the self-diffusion coefficient of HDO in bulk water $\left(\mathrm{D}_{0}\right)$. (a) Pore diffusion coefficients $\left(\mathrm{Dp}_{\mathrm{z}} / \mathrm{D}_{0}\right)$ of water in Na-kaolinite. Experimental data are shown in filled symbols (triangle and diamond for ${ }^{1} \mathrm{H}$ NMR Pulsed Gradient Spin Echo (PGSENMR) and through-diffusion (TD) methods, respectively), whereas simulated data are shown as nonefilled squares. (b) Pore diffusion coefficients $\left(\mathrm{Dp}_{\mathrm{z}} / \mathrm{D}_{0}\right)$ of water in Na-vermiculite. Experimental data are shown in filled symbols (circle and square for the centrifuged and compacted samples, respectively), whereas simulated data are shown as none-filled diamonds. (c) Summary of (a) and (b) displayed as effective diffusion coefficients $\left(\mathrm{De}_{\mathrm{z}} / \mathrm{D}_{0}\right)$. Data are also compared to experimental ( + and $\times$ symbols) and simulated (none-filled circles) results from Dabat et al. (2020). 
3.4. The influence of interlayer vs. interparticle porosities on HDO diffusion in clayey

545

546

547

548

549

550

551

552

553

554

555

556

557

558

559

560

561

562

563

564

565 samples

To assess the role played by the two porosities types (interlayer and interparticle), the HDO diffusivity is discussed below in terms of $D e_{\mathrm{Z}}$ since this parameter is commonly used over $D p_{z}$ in macroscopic models to describe the diffusing flux traversing a sample (Bourg and Tournassat, 2015; Tournassat and Steefel, 2019 and references therein). The obtained $D e_{\mathrm{z}}$ values for water, normalized to self-diffusion in bulk $D_{0}$, measured for both materials (kaolinite and Na-vermiculite) using the two techniques (PSGE-NMR and TD), are plotted in Fig. 5c as a function of the $\left\langle\mathrm{P}_{2}\right\rangle$ parameter. These data are compared to the calculated ones obtained from BD simulations assuming that all porosity is accessible to diffusion (as confirmed by HDO alpha values previously discussed and reported in Table 1). Considering that experimental samples composed of Na-vermiculite and Na-kaolinite particles have approximatively the same interparticle porosity (i.e., 0.26 vs. 0.24 for kaolinite and vermiculite, respectively) and that all of the porosity of the Na-vermiculite samples is accessible to water diffusion, one can expect that $D e_{\mathrm{z}}$ obtained with Na-vermiculite samples are intermediate between those simulated for $\varepsilon_{\text {interp. }}=0.25$ and $\varepsilon_{\text {interp. }}=0.5$ (for similar $\left\langle\mathrm{P}_{2}\right\rangle$ values). In fact, experimental data obtained with Na-vermiculite $(\varepsilon=0.49)$ are drastically much closer to those simulated at $\varepsilon_{\text {interp. }}=0.25$ than to those simulated at $\varepsilon_{\text {interp. }}=0.5$ (Fig. $5 \mathrm{c}$ ). Such behavior suggests that even though interlayer porosity is available for diffusion, the contribution of the flux of HDO diffusing in this interlayer volume is very marginal compared to that in the interparticle porosity, which results in $D e_{\mathrm{Z}}$ values that are almost independent of the interlayer porosity of the material. Interestingly, the evolution of the diffusion anisotropy ratios $\frac{D e_{\overline{x y}}}{D e_{z}}$ with the $\left\langle\mathrm{P}_{2}\right\rangle$ values reported in Fig. 6 indicates a significant increase in anisotropy with the increased degree of preferred orientation of particles. This behavior is the same for different values of the total porosity and different 


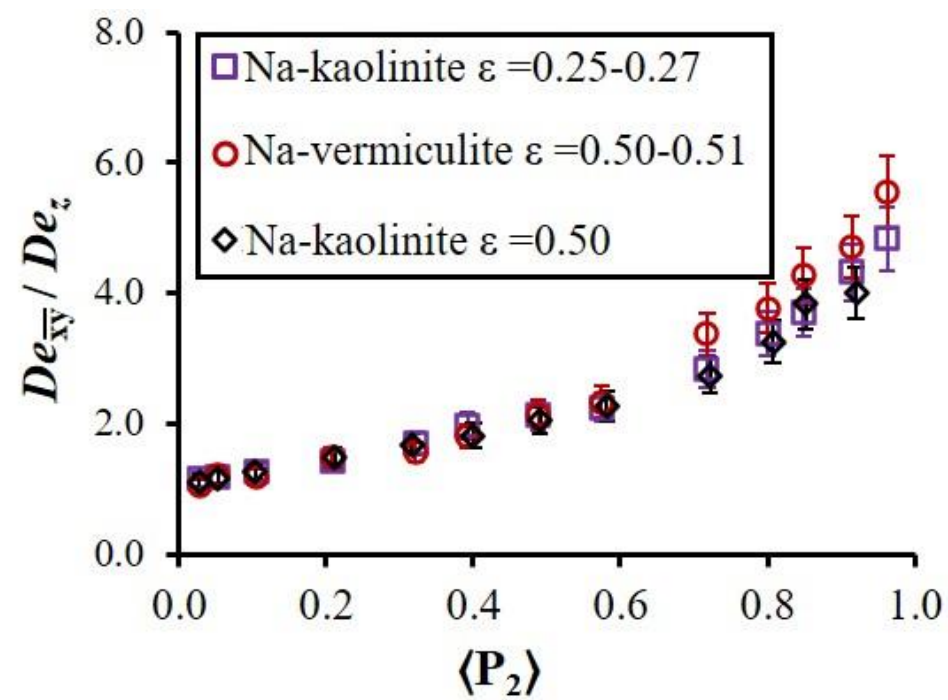

Fig. 6. Influence of porosity and its distribution between interlayer and interparticle volumes on the evolution of the diffusion anisotropy ratio $\mathrm{De}-\overline{\mathrm{xy}} / \mathrm{De}_{\mathrm{z}}$ as a function of the order parameter $\left\langle\mathrm{P}_{2}\right\rangle$. The anisotropy ratio is calculated based on the $\mathrm{BD}$ simulated mean effective water diffusion coefficients in the $(\mathrm{x}, \mathrm{y})$

591 plane $\left(\mathrm{De}_{\overline{\mathrm{xy}}}\right)$ over the $\mathrm{z}$ direction $\left(\mathrm{De}_{\mathrm{z}}\right)$ with respect to the direction of the preferred orientation of the

592 clay particles. Data are shown for Na-kaolinite with $\varepsilon=0.25$ (squares, this study) or $\varepsilon=0.50$ (diamonds,

593 Dabat et al. (2020)), and for Na-vermiculite with $\varepsilon=0.50-0.51$ and $\varepsilon_{\text {interp. }}=0.25-0.27$ (circles, this study). 
595

596

597

598

599

600

601

602

603

604

605

606

607

608

609

610

611

612

613

614

615

616

617

value is not easy for smectite due to osmotic swelling of this mineral, especially when the saturating cations are $\mathrm{Na}^{+}$, which makes it difficult to discriminate the role played by interlayer and interparticle porosities on the global diffusivity of water tracer in this mineral. Such a phenomenon was, to some extent, indicated by Melkior et al. (2009), who reported that the effective diffusion coefficient of water tracer was significantly dependent on the nature of the compensating cations in samples made of smectitic particles (for the same total porosity), with $D e_{\mathrm{z}}$ increasing from Na-smectite, Ca-smectite to Cs-smectite. These authors attributed this variation to the absence of gel formation for the Cs-samples compared to the Na-samples (and to a lesser extent in Ca-samples). This observation was most likely related to the difficulty of considering a defined interparticle porosity (i.e., pores not submitted to the particle charge) for Na-smectites compared to Cs ones, and consequently raises the question about the relevance of considering the $D e_{\mathrm{z}}$ values obtained with Na-smectites as reference data for models describing dual porosity clayey system. In that regard, the experimental results obtained in this study with Na-vermiculite samples represent a new set of data for an extensive validation of dual-porosity reactive transport models.

\section{Concluding remarks and perspectives}

A methodology was developed in this study to prepare experimental clayey samples for which the porosity distribution (interlayer $\left(\varepsilon_{\text {interl. }}\right) /$ interparticle $\left(\varepsilon_{\text {interp. }}\right)$ and particle organization can be controlled independently. These new procedures were successfully applied to prepare samples characterized by the same interparticle porosity but with the presence or not of interlayer porosity accessible to water diffusion (Na-kaolinite $v s$ Na-vermiculite samples), used to investigate the role played by the two types of porosities and the particle organization on the global diffusion of water. 
The similarity of the diffusion data obtained for Na-kaolinite by two independent experimental techniques (i.e., Pulsed Gradient Spin Echo attenuation measurements by Nuclear Magnetic Resonance (PGSE-NMR) and through-diffusion (TD)) probing different length and time scales (i.e., ms for PGSE-NMR vs. several days for TD), shows that a representative elementary volume for the analysis of water diffusion process in clayey materials was reached with PGSE-NMR method. Moreover, these two experimental techniques are found to be very complementary for the analysis of water diffusion in clayey media. On the one hand, PGSENMR allows obtaining the full tensor of the water diffusion on the same sample, whereas the TD experiments must be repeated when changing the probed direction of diffusion. On the other hand, PGSE-NMR cannot be used when the sample contains a very high quantity of paramagnetic elements, such as Fe in the Na-vermiculite used in this study, or when the probe confinement is high (the case of water molecule in the interlayer of Na-vermiculite as a result of its high structural charge). In that regard TD can be advantageously used irrespective of the chemical composition of the sample and interlayer probe confinement.

By accounting for the uncertainties in both the porosity and particle organization measurements, there were good agreements between the effective diffusion coefficients derived from the experimental measurements obtained for all samples and simulated data. This finding showed that, in a dual-porosity clayey media, even though the interlayer is accessible to water diffusion, interparticle porosity and preferential orientation of the particles act as first-order parameters in the global flux of water diffusing through these media, at least for the range of porosity investigated. These results were obtained irrespective of the directional diffusion probed, and they allowed us to conclude that the $\left\langle\mathrm{P}_{2}\right\rangle$ parameter (i.e., mathematical function derived from two-dimensional X-Ray Scattering measurements comprised between 0 for an isotropic particle organization and 1 for a perfectly anisotropic organization) is an excellent 
descriptor of the anisotropy of the diffusion of water in materials made of swelling clay particles.

Due to osmotic swelling of montmorillonite clay minerals, especially when the charge compensating cation is $\mathrm{Na}^{+}$, distribution of interparticle and interlayer porosities is extremely difficult to be determined. As logical continuation of this work, Na-vermiculite samples characterized by well-defined $\varepsilon_{\text {interl }} / \varepsilon_{\text {interp. }}$ ratios and particle organization, can be used for further investigating the link between geometrical properties and the diffusion of charged (ionic) tracers in swelling clays. Such approach would likely provide key information for decoupling the role played by interfacial charges located on either basal or edge surfaces of Navermiculite from other parameters linked to the geometric organization of the particles, such as the preferential orientation of the clay particles or the different porosities accessible to diffusion.

\section{Acknowledgments}

The results presented are part of the Ph.D. thesis of A.A. granted by "Région NouvelleAquitaine", University of Poitiers, France. Stephan Rouzière (LPS, Saclay, France) is acknowledged for technical assistance in the XRS measurements. Dr. Jerome Labanowski (IC2MP, Poitiers, France) is thanked for providing access to the lyophilizer used in this study. Claude Veit (IC2MP, Poitiers, France) is thanked for the design and conception of the compaction and centrifugation cells for the sample preparation. The authors are grateful to the CNRS interdisciplinary "défi Needs" through its "MiPor" program (Project DARIUS), the H2020 European program EURAD through its "Future” WP, the French government program "Investissements d'Avenir" (EUR INTREE, reference ANR-18-EURE-0010), and the European Union (ERDF) and "Région Nouvelle Aquitaine" for providing financial support for this study. 
668

669

670

671

672

673

674

675

676

677

678

679

680

681

682

683

684

685

686

687

688

689

690

691

692

693

694

695

696

697

698

699

700

701

702

703

704

705

706

707

708

709

710

711

712

Abend, S., Lagaly, G., 2000. Sol-gel transitions of sodium montmorillonite dispersions. Applied Clay Science 16, 201-227. https://doi.org/10.1016/S0169-1317(99)00040-X Altmann, S., Aertsens, M., Appelo, T., Bruggeman, C., Gaboreau, S., Glaus, M., Jacquier, P., Kupcik, T., Maes, N., Montoya, V., Rabung, T., Savoye, S., et al, 2015. Processes of cation migration in clayrocks: Final Scientific Report of the CatClay European Project. CEA.

Andra, 2005. Argile : Évaluation de la Faisabilité du Stockage Géologique en Formation Argileuse. Document du Synthèse, Andra.

Arguelles, A., Leoni, M., Blanco, J.A., Marcos, C., 2010. Semi-ordered crystalline structure of the Santa Olalla vermiculite inferred from X-ray powder diffraction. American Mineralogist 95, 126-134. https://doi.org/10.2138/am.2010.3249

Bachu, S., 2008. $\mathrm{CO}_{2}$ storage in geological media: Role, means, status and barriers to deployment. Progress in Energy and Combustion Science 34, 254-273. https://doi.org/10.1016/j.pecs.2007.10.001

Bacle, P., Dufrêche, J.-F., Rotenberg, B., Bourg, I.C., Marry, V., 2016. Modeling the transport of water and ionic tracers in a micrometric clay sample. Applied Clay Science 123, 18-28. https://doi.org/10.1016/j.clay.2015.12.014

Bear, J., 1972. Dynamics of Fluids in Porous Media. Dover Publication, New York.

Bestel, M., 2014. Water-montmorillonite systems: Neutron scattering and tracer throughdiffusion studies (Thesis). University of Bern, Bern-Switzerland.

Bestel, M., Glaus, M.A., Frick, S., Gimmi, T., Juranyi, F., Van Loon, L.R., Diamond, L.W., 2018. Combined tracer through-diffusion of HTO and 22Na through Namontmorillonite with different bulk dry densities. Applied Geochemistry 93, 158-166. https://doi.org/10.1016/j.apgeochem.2018.04.008

Bennett, R.H., Brya, W.R., Keller, G.H., 1981. Clay fabric of selected submarine sediments; fundamental properties and models. Journal of Sedimentary Research 51, 217-232. https://doi.org/10.1306/212F7C52-2B24-11D7-8648000102C1865D

Bourg, I.C., Bourg, A.C.M., Sposito, G., 2003. Modeling diffusion and adsorption in compacted bentonite: a critical review. Journal of Contaminant Hydrology 61, 293302. https://doi.org/10.1016/S0169-7722(02)00128-6

Bourg, I.C., Sposito, G., Bourg, A.C.M., 2006. Tracer diffusion in compacted, water-saturated bentonite. Clays Clay Miner. 54, 363-374. https://doi.org/10.1346/CCMN.2006.0540307

Bourg, I.C., Tournassat, C., 2015. Self-Diffusion of Water and Ions in Clay Barriers, in: Developments in Clay Science. Elsevier, pp. 189-226. https://doi.org/10.1016/B9780-08-100027-4.00006-1

Callaghan, P.T., 1991. Principles of nuclear magnetic resonance microscopy. Clarendon Press.

Charlet, L., Alt-Epping, P., Wersin, P., Gilbert, B., 2017. Diffusive transport and reaction in clay rocks: A storage (nuclear waste, $\mathrm{CO}_{2}, \mathrm{H}_{2}$ ), energy (shale gas) and water quality issue. Advances in Water Resources 106, 39-59. https://doi.org/10.1016/j.advwatres.2017.03.019

Churakov, S.V., Gimmi, T., 2011. Up-Scaling of Molecular Diffusion Coefficients in Clays: A Two-Step Approach. J. Phys. Chem. C 115, 6703-6714. https://doi.org/10.1021/jp112325n 
Dabat, T., Hubert, F., Paineau, E., Launois, P., Laforest, C., Grégoire, B., Dazas, B., Tertre, E., Delville, A., Ferrage, E., 2019. A general orientation distribution function for clayrich media. Nat Commun 10, 5456. https://doi.org/10.1038/s41467-019-13401-0

Dabat, T., Mazurier, A., Hubert, F., Tertre, E., Grégoire, B., Dazas, B., Ferrage, E., 2018. Mesoscale Anisotropy in Porous Media Made of Clay Minerals. A Numerical Study Constrained by Experimental Data. Materials 11, 1972. https://doi.org/10.3390/ma11101972

Dabat, T., Porion, P., Hubert, F., Paineau, E., Dazas, B., Grégoire, B., Tertre, E., Delville, A., Ferrage, E., 2020. Influence of preferred orientation of clay particles on the diffusion of water in kaolinite porous media at constant porosity. Applied Clay Science 184, 105354. https://doi.org/10.1016/j.clay.2019.105354

Dagnelie, R.V.H., Arnoux, P., Enaux, J., Radwan, J., Ner, P., 2017. Perturbation induced by a nitrate plume on diffusion of solutes in a large-scale clay rock sample. Applied Clay Science 141. 219-226.

Dagnelie, R.V.H., Rasamimanana, S., Blin, V., Radwan, J., Thory, E., Robinet, J.-C., Lefèvre, G., 2018. Diffusion of organic anions in clay-rich media: Retardation and effect of anion exclusion. Chemosphere 213, 472-480. https://doi.org/10.1016/j.chemosphere.2018.09.064

De La Calle, C., Pezerat, H., Gasperin, M., 1977. Problemes d'ordre-désordre dans les vermiculites structure du minéral calcique hydraté a 2 couches. Journal de Physique $38,128-133$.

Durrieu, J., Argillier, J.F., Rosenberg, E., Li, Y., 1997. Static and Dynamic Filtration Properties of Aqueous Suspensions of Clays and Electrolytes. Rev. Inst. Fr. Pét. 52, 207-218. https://doi.org/10.2516/ogst:1997020

Dykhuizen, R.C., Casey, W.H., 1989. An analysis of solute diffusion in rocks. Geochimica et Cosmochimica Acta 53, 2797-2805. https://doi.org/10.1016/0016-7037(89)90157-9

Farver, J.R., Yund, R.A., 1999. Oxygen bulk diffusion measurements and TEM characterization of a natural ultramylonite: implications for fluid transport in micabearing rocks. J. Metamorph. Geol 17, 669-683.

Faurel, M., 2012. Conception et mise en place d'expériences de diffusion de l'eau et de solutés dans des milieux poreux modèles d'argiles gonflantes. Université de Poitiers, Poitiers, France.

Ferrage, E., Hubert, F., Baronnet, A., Grauby, O., Tertre, E., Delville, A., Bihannic, I., Prêt, D., Michot, L.J., Levitz, P., 2018. Influence of crystal structure defects on the smallangle neutron scattering/diffraction patterns of clay-rich porous media. $\mathrm{J}$ Appl Crystallogr 51, 1311-1322. https://doi.org/10.1107/S160057671801052X

Ferrage, E., Hubert, F., Tertre, E., Delville, A., Michot, L.J., Levitz, P., 2015. Modeling the arrangement of particles in natural swelling-clay porous media using threedimensional packing of elliptic disks. Physical Review E 91, 062210. https://doi.org/10.1103/PhysRevE.91.062210

García-Gutiérrez, M., Cormenzana, J.L., Missana, T., Mingarro, M., Martín, P.L., 2006. Large-scale laboratory diffusion experiments in clay rocks. Physics and Chemistry of the Earth, Parts A/B/C 31, 523-530. https://doi.org/10.1016/j.pce.2006.04.004

Gieseking, J.E. (Ed.), 1975. Soil Components Vol. 2: Inorganic Components, 1st ed. SpringerVerlag Berlin Heidelberg.

Gimmi, T., Churakov, S.V., 2019. Water retention and diffusion in unsaturated clays: Connecting atomistic and pore scale simulations. Applied Clay Science 175, 169-183. https://doi.org/10.1016/j.clay.2019.03.035 
Gimmi, T., Kosakowski, G., 2011. How Mobile Are Sorbed Cations in Clays and Clay Rocks? Environ. Sci. Technol. 45, 1443-1449. https://doi.org/10.1021/es1027794

Glaus, M.A., Frick, S., Rossé, R., Loon, L.R.V., 2010. Comparative study of tracer diffusion of $\mathrm{HTO},{ }^{22} \mathrm{Na}^{+}$and ${ }^{36} \mathrm{Cl}^{-}$in compacted kaolinite, illite and montmorillonite. Geochimica et Cosmochimica Acta 74, 1999-2010. https://doi.org/10.1016/j.gca.2010.01.010

Gonzáles García, F., García Ramos, G., 1960. On the genesis and transformation of vermiculite. Presented at the Transactions 7th int. Congr. Soil Sci., Spain, pp. 482491.

González Sánchez, F., Jurányi, F., Gimmi, T., Van Loon, L., Unruh, T., Diamond, L.W., 2008a. Translational diffusion of water and its dependence on temperature in charged and uncharged clays: A neutron scattering study. J. Chem. Phys. 129, 174706. https://doi.org/10.1063/1.3000638

González Sánchez, F., Van Loon, L.R., Gimmi, T., Jakob, A., Glaus, M.A., Diamond, L.W., 2008b. Self-diffusion of water and its dependence on temperature and ionic strength in highly compacted montmorillonite, illite and kaolinite. Applied Geochemistry 23, 3840-3851. https://doi.org/10.1016/j.apgeochem.2008.08.008

Gunsteren, W.F., Berendsen, H.J.C., Rullmann, J.A.C., 1981. Stochastic dynamics for molecules with constraints. Molecular Physics 44, 69-95. https://doi.org/10.1080/00268978100102291

Hassan, M.S., Villieras, F., Gaboriaud, F., Razafitianamaharavo, A., 2005. AFM and lowpressure argon adsorption analysis of geometrical properties of phyllosilicates. Journal of Colloid and Interface Science 296, 614-623. https://doi.org/10.1016/j.jcis.2005.09.028

Hermans, P.H., Platzek, P., 1939. Beiträge zur Kenntnis des Deformationsmechanismus und der Feinstruktur der Hydratzellulose. Kolloid-Z, Kolloid-Z 88, 68-72.

Jacops, E., Aertsens, M., Maes, N., Bruggeman, C., Swennen, R., Krooss, B., AmannHildenbrand, A., Littke, R., 2017. The Dependency of Diffusion Coefficients and Geometric Factor on the Size of the Diffusing Molecule: Observations for Different Clay-Based Materials. Geofluids 2017, 1-16. https://doi.org/10.1155/2017/8652560

Landais, P., 2006. Advances in geochemical research for the underground disposal of highlevel, long-lived radioactive waste in a clay formation. Journal of Geochemical Exploration 88, 32-36. https://doi.org/10.1016/j.gexplo.2005.08.011

Liu, L., 2013. Prediction of swelling pressures of different types of bentonite in dilute solutions. Colloids and Surfaces A: Physicochemical and Engineering Aspects 434, 303-318. https://doi.org/10.1016/j.colsurfa.2013.05.068

Loeber, L., 1992. Etude de la structure des cakes d'argile formés sur la paroi des puits au cours du forage. Université d'Orléans, Orléans-France.

Malikova, N., Cadéne, A., Marry, V., Dubois, E., Turq, P., Zanotti, J.-M., Longeville, S., 2005. Diffusion of water in clays - microscopic simulation and neutron scattering. Chemical Physics 317, 226-235. https://doi.org/10.1016/j.chemphys.2005.04.035

Malikova, N., Cadène, A., Marry, V., Dubois, E., Turq, P., 2006. Diffusion of Water in Clays on the Microscopic Scale: Modeling and Experiment. J. Phys. Chem. B 110, 32063214. https://doi.org/10.1021/jp056954z

Marcos, C., Argüelles, A., Ruíz-Conde, A., P. J. Sánchez-Soto, Blanco, J.A., 2003. Study of the dehydration process of vermiculites by applying a vacuum pressure: formation of interstratified phases. Mineral. mag. 67, 1253-1268. https://doi.org/10.1180/0026461036760163 
Marry, V., Turq, P., Cartailler, T., Levesque, D., 2002. Microscopic simulation of structure and dynamics of water and counterions in a monohydrated montmorillonite. The Journal of Chemical Physics 117, 3454-3463. https://doi.org/10.1063/1.1493186

Marry, V., Turq, P., 2003. Microscopic Simulations of Interlayer Structure and Dynamics in Bihydrated Heteroionic Montmorillonites. J. Phys. Chem. B 107, 1832-1839.

Melkior, T., Gaucher, E.C., Brouard, C., Yahiaoui, S., Thoby, D., Clinard, Ch., Ferrage, E., Guyonnet, D., Tournassat, C., Coelho, D., 2009. $\mathrm{Na}^{+}$and HTO diffusion in compacted bentonite: Effect of surface chemistry and related texture. Journal of Hydrology 370, 9-20. https://doi.org/10.1016/j.jhydrol.2009.02.035

Mermut, A.R., Cano, A.F., 2001. Baseline Studies of the Clay Minerals Society Source Clays: Chemical Analyses of Major Elements. Clays and Clay Minerals 49, 381-386. https://doi.org/10.1346/CCMN.2001.0490504

Michot, L.J., Ferrage, E., Jiménez-Ruiz, M., Boehm, M., Delville, A., 2012. Anisotropic Features of Water and Ion Dynamics in Synthetic Na- and Ca-Smectites with Tetrahedral Layer Charge. A Combined Quasi-elastic Neutron-Scattering and Molecular Dynamics Simulations Study. J. Phys. Chem. C 116, 16619-16633. https://doi.org/10.1021/jp304715m

Nakashima, Y., Mitsumori, F., 2005. $\mathrm{H}_{2} \mathrm{O}$ self-diffusion restricted by clay platelets with immobilized bound $\mathrm{H} 2 \mathrm{O}$ layers: PGSE NMR study of water-rich saponite gels. Applied Clay Science 28, 209-221. https://doi.org/10.1016/j.clay.2004.01.017

Ochs, M., Lothenbach, B., Wanner, H., Sato, H., Yui, M., 2001. An integrated sorptiondiffusion model for the calculation of consistent distribution and diffusion coefficients in compacted bentonite. Journal of Contaminant Hydrology 47, 283-296. https://doi.org/10.1016/S0169-7722(00)00157-1

Porion, P., Faugère, A.M., Delville, A., 2014. Structural and Dynamical Properties of Water Molecules Confined within Clay Sediments Probed by Deuterium NMR Spectroscopy, Multiquanta Relaxometry, and Two-Time Stimulated Echo Attenuation. J. Phys. Chem. C 118, 20429-20444. https://doi.org/10.1021/jp506312q

Porion, P., Ferrage, E., Hubert, F., Tertre, E., Dabat, T., Faugère, A.M., Condé, F., Warmont, F., Delville, A., 2018. Water Mobility within Compacted Clay Samples: Multi-Scale Analysis Exploiting ${ }^{1}$ H NMR Pulsed Gradient Spin Echo and Magnetic Resonance Imaging of Water Density Profiles. ACS Omega 3, 7399-7406. https://doi.org/10.1021/acsomega.8b01083

Pret, D., 2003. Nouvelles méthodes quantitatives de cartographie de la minéralogie et de la porosité dans les matériaux argileux : application aux bentonites compactées des barrières ouvragées. Université de Poitiers, Poitiers-France.

Pusch, R., 1979. Highly Compacted Sodium Bentonite for Isolating Rock-Deposited Radioactive Waste Products. Journal Nuclear Technology 45, 153-157. https://doi.org/10.13182/NT79-A32305

Reinholdt, M.X., Hubert, F., Faurel, M., Tertre, E., Razafitianamaharavo, A., Francius, G., Prêt, D., Petit, S., Béré, E., Pelletier, M., Ferrage, E., 2013. Morphological properties of vermiculite particles in size-selected fractions obtained by sonication. Applied Clay Science 77-78, 18-32. https://doi.org/10.1016/j.clay.2013.03.013

Sakharov, B.A., Drits, V.A., McCarty, D.K., Walker, G.M., 2016. Modeling Powder X-Ray Diffraction Patterns of the Clay Minerals Society Kaolinite Standards: Kga-1, Kga-1b, and Kga-2. Clays Clay Miner. 64, 314-333. https://doi.org/10.1346/CCMN.2016.0640307

Sato, H., 2000. The Effect of Pore Structural Factors on Diffusion in Compacted Sodium Bentonite. MRS Proc. 663, 605. https://doi.org/10.1557/PROC-663-605 
Sato, H., Suzuki, S., 2003. Fundamental study on the effect of an orientation of clay particles on diffusion pathway in compacted bentonite. Applied Clay Science 23, 51-60. https://doi.org/10.1016/S0169-1317(03)00086-3

Savoye, S., Beaucaire, C., Grenut, B., Fayette, A., 2015. Impact of the solution ionic strength on strontium diffusion through the Callovo-Oxfordian clayrocks: An experimental and modeling study. Applied Geochemistry 61, 41-52. https://doi.org/10.1016/j.apgeochem.2015.05.011

Shackelford, C.D., Moore, S.M., 2013. Fickian diffusion of radionuclides for engineered containment barriers: Diffusion coefficients, porosities, and complicating issues. Engineering Geology 152, 133-147. https://doi.org/10.1016/j.enggeo.2012.10.014

Skipper, N.T., Lock, P.A., Titiloye, J.O., Swenson, J., Mirza, Z.A., Howells, W.S., Fernandez-Alonso, F., 2006. The structure and dynamics of 2-dimensional fluids in swelling clays. Chemical Geology 230, 182-196. https://doi.org/10.1016/j.chemgeo.2006.02.023

Suzuki, S., Sato, H., Ishidera, T., Fujii, N., 2004. Study on anisotropy of effective diffusion coefficient and activation energy for deuterated water in compacted sodium bentonite. Journal of Contaminant Hydrology 68, 23-37. https://doi.org/10.1016/S01697722(03)00139-6

Swenson, J., Bergman, R., Howells, W.S., 2000. Quasielastic neutron scattering of twodimensional water in a vermiculite clay. J. Chem. Phys. 113, 2873-2879. https://doi.org/10.1063/1.1305870

Tertre, E., Delville, A., Prêt, D., Hubert, F., Ferrage, E., 2015. Cation diffusion in the interlayer space of swelling clay minerals - A combined macroscopic and microscopic study. Geochimica et Cosmochimica Acta 149, 251-267. https://doi.org/10.1016/j.gca.2014.10.011

Tertre, E., Savoye, S., Hubert, F., Prêt, D., Dabat, T., Ferrage, E., 2018. Diffusion of Water through the Dual-Porosity Swelling Clay Mineral Vermiculite. Environ. Sci. Technol. 52, 1899-1907. https://doi.org/10.1021/acs.est.7b05343

Titiloye, J.O., Skipper, N.T., 2001. Molecular dynamics simulation of methane in sodium montmorillonite clay hydrates at elevated pressures and temperatures. Molecular Physics 99, 899-906. https://doi.org/10.1080/00268970010028863

Tournassat, C., Steefel, C.I., 2019. Reactive Transport Modeling of Coupled Processes in Nanoporous Media. Reviews in Mineralogy and Geochemistry 85, 75-109. https://doi.org/10.2138/rmg.2019.85.4

Tyagi, M., Gimmi, T., Churakov, S.V., 2013. Multi-scale micro-structure generation strategy for up-scaling transport in clays. Advances in Water Resources 59, 181-195. https://doi.org/10.1016/j.advwatres.2013.06.002

Underwood, T.R., Bourg, I.C., 2020. Large-Scale Molecular Dynamics Simulation of the Dehydration of a Suspension of Smectite Clay Nanoparticles. J. Phys. Chem. C 124, 3702-3714. https://doi.org/10.1021/acs.jpcc.9b11197

Valleau, J., Diestler, D., Cushman, J., Schoen, M., Hertzner, A., Riley, M., 1991. Comment on: Adsorption and diffusion at rough surfaces. A comparison of statistical mechanics, molecular dynamics, and kinetic theory. The Journal of Chemical Physics 95, 61946195.

Van Loon, L.R., Soler, J.M., Jakob, A., Bradbury, M.H., 2003. Effect of confining pressure on the diffusion of $\mathrm{HTO},{ }^{36} \mathrm{Cl}^{-}$and ${ }^{125} \mathrm{I}^{-}$in a layered argillaceous rock (Opalinus Clay): diffusion perpendicular to the fabric. Applied Geochemistry 18, 1653-1662. https://doi.org/10.1016/S0883-2927(03)00047-7 
Van Loon, L.R., Soler, J.M., Müller, W., Bradbury, M.H., 2004. Anisotropic Diffusion in Layered Argillaceous Rocks: A Case Study with Opalinus Clay. Environ. Sci. Technol. 38, 5721-5728.

Wenk, H.-R., Kanitpanyacharoen, W., Voltolini, M., 2010. Preferred orientation of phyllosilicates: Comparison of fault gouge, shale and schist. Journal of Structural Geology 12, 478-489.

Wenk, Voltolini, Mazurek, Loon, V.L.R., Vinsot, 2008. Preferred Orientations and Anisotropy in Shales: Callovo-Oxfordian Shale (France) and Opalinus Clay (Switzerland). Clays Clay Miner. 56, 285-306. https://doi.org/10.1346/CCMN.2008.0560301

Wittebroodt, C., Savoye, S., Frasca, B., Gouze, P., 2012. Diffusion of HTO, ${ }^{36} \mathrm{Cl}^{-}$and ${ }^{125} \mathrm{I}^{-}$in Upper Toarcian argillite samples from Tournemire: Effects of initial iodide concentration and ionic strength. Applied Geochemistry 27, 1432-1441.

Xiang, Y., Al, T., Scott, L., Loomer, D., 2013. Diffusive anisotropy in low-permeability Ordovician sedimentary rocks from the Michigan Basin in southwest Ontario. Journal of Contaminant Hydrology 155, 31-45.

\section{Supplementary data (S. D.)}

\section{S.1. Materials and methods}

\section{S.1.1. ${ }^{1}$ H NMR Pulsed Gradient Spin Echo experiments}

The Pulsed Gradient Spin Echo attenuation measurements by Nuclear Magnetic Resonance (PGSE-NMR) analyses were performed at the ICMN laboratory (Orléans, France) on the Na-kaolinite compacted sample. The dried Na-kaolinite samples, in volume-constrained Polyether ether ketone (PEEK) tubes (see Section S.1.1), were first water-saturated by capillary imbibition. To do so, the bottom part of the sample was put in contact with deionized water, while the other side of the tube was connected to a primary vacuum pump to favor water migration and gentle evacuation of air from the pore network. The NMR measurements were performed using the same setup and experimental conditions as in (Porion et al., 2018; Dabat et al., 2020) on a Bruker ${ }^{\circledR}$ DSX100 spectrometer. This instrument operates with a static magnetic field of $2.35 \mathrm{~T}$ and is equipped with a saddle detection coil and a micro-imaging probe (Micro5 Bruker) with gradient coils to generate magnetic field gradients in three perpendicular directions. The macroscopic water mobility along any pre-selected direction within the sample 
was determined based on ${ }^{1} \mathrm{H}$ PGSE-NMR attenuation measurements (Stejskal and Tanner, 1965; Cotts et al., 1989; Callaghan, 1991) by using the pulse sequence illustrated in Fig. S1. The largest wave vector ( $\left.\mathrm{q}_{\mathrm{MAX}}\right)$ associated with these attenuation measurements is defined as $\mathrm{qMAX}_{\mathrm{M}}=\frac{\gamma \cdot \delta \cdot G_{M A X}}{\pi}=6.8110^{4} \mathrm{~m}^{-1}$, where $\gamma$ is the proton gyromagnetic ratio $\left(2.675210^{8} \mathrm{read} / \mathrm{s}\right.$ for ${ }^{1} \mathrm{H}$ ), $\delta$ is the duration of each applied field gradient (i.e., $0.5 \mathrm{~ms}$ ) (see Fig. S1) and GMAX is the largest applied field gradient (i.e., 1.6 Tesla $\mathrm{m}^{-1}$ ). Accordingly, the spatial length over which the dynamics of the water is investigated is larger than $15 \mu \mathrm{m}$. For these experiments, the diffusion time $(\Delta)$ and the delay $(\tau)$ were set to $20 \mathrm{~ms}$ and $760 \mu$ s, respectively (Fig. S1). The attenuation of the intensity of the NMR echo $I(q) / I(0)$ evolves according to a Gaussian relationship from which the components of the self-diffusion tensor are easily extracted (Cotts et al., 1989; Callaghan, 1991):

$$
\frac{I(q)}{I(0)}=\exp \left[-4 \pi^{2} q^{2}{\overrightarrow{e_{g}}}^{T} \stackrel{D p}{e_{g}}(\Delta+3 \tau / 2-\delta / 6)\right]
$$

where $\overrightarrow{e_{g}}$ is the direction of the applied field gradient, and $\overleftrightarrow{D p}$ is the self-diffusion tensor. The pulsed gradients were applied along six non-collinear directions (Basser et al., 1994; Skare et al., 2000) noted $\overrightarrow{e_{1}}=\overrightarrow{e_{x}}=(1,0,0), \quad \overrightarrow{e_{2}}=\overrightarrow{e_{y}}=(0,1,0), \quad \overrightarrow{e_{3}}=\overrightarrow{e_{z}}=(0,0,1), \overrightarrow{e_{4}}=$ $(1 / \sqrt{2}, 1 / \sqrt{2}, 0), \overrightarrow{e_{5}}=(0,1 / \sqrt{2}, 1 / \sqrt{2})$ and $\overrightarrow{e_{6}}=(1 / \sqrt{2}, 0,1 / \sqrt{2})$, respectively, with the direction $\overrightarrow{e_{3}}$ being parallel to the compaction/centrifugation axis of the clay sample. From the six corresponding components of the water self-diffusion tensor, one can easily extract its three principal axes and the corresponding eigenvalues that describe the water mobility in the longitudinal or transverse direction of the tube axis. The PGSE-NMR attenuation measurements were performed at $\sim 19^{\circ} \mathrm{C}$ (i.e., $292 \mathrm{~K}$ ), and the associated experimental bulk water selfdiffusion coefficient $D_{0}$ was measured at $2.0 \pm 0.5 .10^{-9} \mathrm{~m}^{2} \mathrm{~s}^{-1}$ in these conditions. 


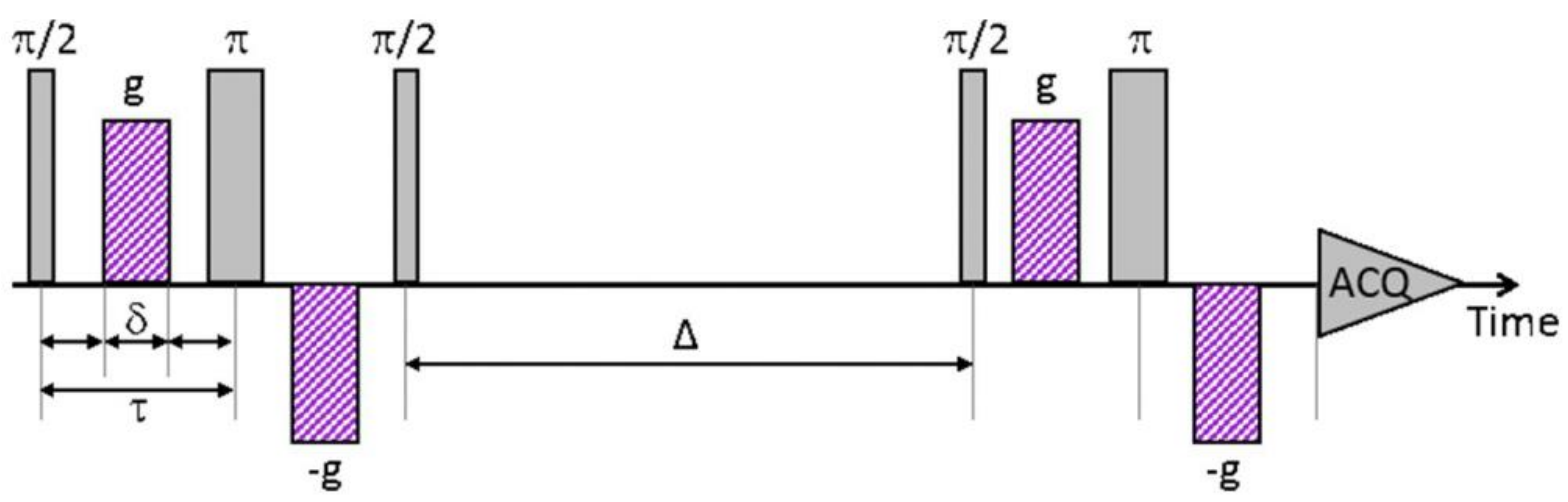

960

961

962

963

964

965

966

967

968

969

970

971

972

973

974

975

976

977

978

979

980

Fig. S1. Pulse sequence used to perform Pulsed Gradient Spin Echo (PGSE-NMR) attenuation measurements in Na-kaolinite samples.

\section{. Induration of the samples and two-dimensional X-Ray Scattering measurements}

For each sample used in this study, a duplicate was prepared to be used for particle organization measurements. Induration of the duplicate samples was performed to produce thin sections for two-dimensional X-Ray Scattering (2D-XRS) measurements. The induration method used here was based on the thermal impregnation technique with methyl methacrylate (MMA, $\mathrm{C}_{5} \mathrm{H}_{8} \mathrm{O}_{2}$ ), which was developed by Sammaljärvi et al. (2012) and successfully applied for Na-kaolinite samples (Dabat et al., 2020). The duplicates for 2D-XRS were prepared in PEEK tubes (diameter of $0.64 \mathrm{~cm}$ and height of $7.5 \mathrm{~cm}$ ) instead of PMMA because the latter can dissolve when in contact with MMA. Perforated PEEK caps and cellulose membrane with a pore size of $0.1 \mu \mathrm{m}$ were placed at each side to maintain both the total porosity and particle organization of the sample during the induration process. The duplicates were first placed under a vacuum for 20 minutes to remove the residual water that could interfere with the polymerization of MMA. Then, liquid MMA mixed with thermal initiator benzoyl peroxide (BPO, at $5 \%$ of MMA mass) was introduced into the tubes without breaking the vacuum to facilitate the access of MMA gas to the smallest pores. The samples were kept in contact with liquid MMA for a few days up to two weeks (in the case of centrifuged Na-vermiculite) to promote as much as possible the capillary saturation of the pores by the MMA+BPO mixture. 
After saturation, the PEEK tubes that contained clayey samples were sealed with PTFE caps and placed in a water bath at $55^{\circ} \mathrm{C}$ for at least 24 hours to complete the polymerization of MMA into PMMA.

Following the induration process, the obtained samples were cut longitudinally and transversely with respect to the tube axis (Fig. 2a) using a circular saw (Fig. 2b). The resulting lamellas were reduced by a polishing table to $500 \mu \mathrm{m}$ thickness to allow sufficient transmission for the XRS measurements (Dabat et al., 2020). Acquisition of two-dimensional XRS (2DXRS) patterns for anisotropy in particle orientation measurements was performed at the Laboratoire de Physique des Solides (LPS) - Orsay, France. The X-ray beam is generated via a copper rotating anode generator (RU H3R, Rigaku Corporation, Japan), equipped with a multilayer W/Si mirror (Osmic), which generates a monochromatic beam $\left(\lambda_{\mathrm{CuK} \alpha}=1.5418 \AA\right)$ with a spot size of $1 \mathrm{~mm}^{2}$ that passes through the slits to cover a $600 \times 600 \mu \mathrm{m}^{2}$ surface of the sample. The scattering signals (Fig. 2c) from the sample were collected on a 2D-detector (MAR345, marXperts $\mathrm{GmbH}^{\circledR}$, Germany, 150- $\mu \mathrm{m}$ pixel size). The sample-to-detector distance was set to $250 \mathrm{~mm}$. This configuration makes it possible to reach a scattering vector modulus down to $Q_{\min }=0.2 \AA^{-1}\left(Q=2 \pi / d=4 \pi / \lambda \sin \left(\theta_{B}\right)\right.$, where $\lambda$ is the incident wavelength, and $2 \theta_{B}$ is the scattering angle). The sample lamellas were aligned perpendicular to the incident X-ray beam by mounting them on a goniometer head, and the XRS were acquired with a typical acquisition time of $900 \mathrm{~s}$.

\section{S.1.3. Through-diffusion experiments on the HDO tracer}

The experimental setup for the Through-Diffusion (TD) experiments on the water tracer (HDO) was previously used by Tertre et al. (2018) and originally adapted from the one proposed by Van Loon et al. (2003). The configurations of this setup allow for measuring HDO diffusion along the $z$-direction, i.e., perpendicular to the compaction plane and the preferred orientation of the clay particles. It consists of a fluid circulation system and a PMMA tube that contains 
the clay sample compacted at the desired $\varepsilon$ value (see section 2.2 in the original text). The sample is retained in the tube via a series of components on each side, listed from the closest to the sample to the farthest, as follows: (i) cellulose membrane with a pore size of $0.1 \mu \mathrm{m}$, (ii) a stainless-steel filter (pore diameter of $10 \mu \mathrm{m}$ ), and (iii) two PEEK grids (nominal spaces of 280 and $450 \mu \mathrm{m}$ for mono filaments with diameters equal to 120 and $200 \mu \mathrm{m}$, respectively). The PEEK grids are used to homogenize the flow of the solution that arrives at each side of the sample (Melkior, 2000). The fluid circulation system consists of two 50-mL reservoirs (i.e., upstream, and downstream reservoirs) and a peristaltic pump used to ensure the constant circulation of the solutions. Prior to the TD experiment, the sample was saturated with Milli$\mathrm{Q}^{\circledR}$ water $(\sim 18.2 \mathrm{M} \Omega \mathrm{cm})$ by maintaining circulation of the water on each side of it for two weeks (Tertre et al., 2018).

For the TD experiments, the upstream reservoir was filled with $50 \mathrm{~mL}$ of a $0.01-\mathrm{M} \mathrm{NaCl}$ solution, spiked with $\mathrm{HDO}$ at $0.55 \mathrm{M}$ and prepared by a dilution of initial $\mathrm{D}_{2} \mathrm{O}$ solution (purity of 99.8 atom \% D purchased from Agros Organics ${ }^{\circledR}$ ), and the downstream reservoir was filled with $50 \mathrm{~mL}$ of Milli-Q water. The $0.01-\mathrm{M} \mathrm{NaCl}$ solution was used in the upstream reservoir to to measure the in addition to $\mathrm{HDO}$ the diffusion of ions (i.e., $\mathrm{Na}^{+}, \mathrm{Cl}^{-}$) as performed previously in Tertre et al. (2018); data which are not reported in this present study. The two reservoirs were then connected to the TD setup, and $t=0$ was set as the fluid circulation started. To keep the HDO concentration gradient as constant as possible between the two reservoirs, both reservoirs were regularly replaced with fresh ones (the time step can vary between a few hours to 1 day depending on the experiments), and $1 \mathrm{~mL}$ aliquot from the reservoirs were collected for the HDO concentration measurements. The results from the upstream reservoirs (not shown) confirmed that the decrease in the HDO concentration did not exceed $3 \%$, which validated the hypothesis of a constant gradient of HDO during the experiment (see below for the boundary conditions). 
All of the TD experiments were performed in a climate-controlled room under a

1032

1033

1034

1035

1036

1037

1038

1039

1040

1041

1042

1043

1044

1045

1046

1047

1048

1049

1050

1051

1052

1053

1054

temperature of $20 \pm 1{ }^{\circ} \mathrm{C}$. The HDO concentrations were determined by water isotope analysis (LWIA DLT-100, Los Gatos Research), and the amount of HDO diffusing in the downstream reservoir was calculated by accounting for the HDO concentration that was naturally present in ultrapure water (i.e., $1.6529 \times 10^{-2} \mathrm{M}$ ), as performed in Tertre et al. (2018).

The results from the TD experiments were analyzed by resolving the classical Fick's second law for one-dimensional transport:

$$
\frac{\partial C}{\partial t}=\frac{D_{e}}{\alpha} \cdot \frac{\partial^{2} C}{\partial x^{2}}=\frac{D_{e}}{\varepsilon+\rho_{\text {app } K_{\mathrm{d}}}} \frac{\delta^{2} C}{\partial x^{2}}
$$

where $C$ is the aqueous concentration $\left(\mathrm{mol} \mathrm{m}^{-3}\right), t$ is the time (s), De is the effective diffusion coefficient $\left(\mathrm{m}^{2} \mathrm{~s}^{-1}\right), K_{\mathrm{d}}$ is the distribution coefficient $\left(\mathrm{m}^{3} \mathrm{~kg}^{-1}\right), \rho_{\text {app }}$ is the bulk dry density $(\mathrm{kg}$ $\mathrm{m}^{-3}$ ), and $\alpha$ is the rock capacity factor. For the water tracer (-), if no adsorption occurs, then $\alpha$ is equal to $\varepsilon$, while if adsorption occurs, $\alpha$ could be greater than $\varepsilon$. Eq. (S2) was resolved by using the following initial and boundary conditions:

$$
\begin{aligned}
& C(x, t)=0 \text { for } t=0 \\
& C(x, t)=C_{o} \text { at } x=0 \text { for } t>0 \\
& C(x, t)=0 \text { at } x=L \text { for } t>0
\end{aligned}
$$

where $C_{o}$ is the HDO concentration in the upstream reservoir $\left(\mathrm{mol} \mathrm{m}^{-3}\right)$ corrected from the natural concentration of HDO present in ultra-pure water, and $L$ is the thickness of the sample (m). By considering these equations, the diffusive flux (i.e., $J(x=L, t)$ in $m o l \mathrm{~s}^{-1}$ ) in the downstream reservoir can be described according to Eq. (S6), as reported by Crank (1975):

$$
J(x=L, t)=\frac{S C_{0} D_{e}}{L}\left(1+2 \sum_{n=1}^{\infty}(-1)^{n} \exp \left(\frac{-D_{e} n^{2} \pi^{2} t}{\alpha L^{2}}\right)\right)
$$

where $S$ is the cross-sectional area perpendicular to the diffusive direction $\left(\mathrm{m}^{2}\right)$. The corresponding total amount of cumulative tracer in the downstream reservoir (i.e., $n(x=L, t)$ in mol) is as follows: 


$$
n(x=L, t)=S C_{O} L\left(\frac{D_{e}}{L^{2}} t-\frac{\alpha}{6}-\frac{2 \alpha}{\pi^{2}} \sum_{n=1}^{\infty} \frac{(-1)^{n}}{n^{2}} \exp \left(-\frac{D_{e} n^{2} \pi^{2}}{L^{2}} t\right)\right.
$$

As done in Tertre et al. (2018), the diffusion parameters (i.e., De and $\alpha$ ) were obtained

1057

1058

1059

1060

1061

1062

1063

1064

1065

1066

1067

1068

1069

1070

1071

1072

1073

1074

1075

1076

1077

1078

1079

by least-square fitting of the experimental results of the diffusive flux incoming in the downstream reservoir. To accomplish that step, fully analytical solutions were obtained in Laplace space and were then subsequently numerically inverted to provide the solution in time (more details in Savoye et al., 2015). Fitting procedures were performed by accounting for diffusion in stainless-steel filters, as performed in Tertre et al. (2018). Uncertainties in both $D_{e}$ and $\alpha$ were calculated by considering the uncertainties in the measured tracer fluxes (corresponding to the uncertainty in the concentration measurements; see Table 1).

\section{S.1.4. Generation of virtual porous media for Brownian dynamics simulation}

Mesoscale Brownian dynamics simulations of water diffusion in both Na-vermiculite and Na-kaolinite as a function of the anisotropy in the particles' orientation were performed on 3D virtual porous media (VPM; see Table S1), mimicking the distribution of the shapes and sizes of the particles in these samples. The description of the generation of these VPM has been extensively described elsewhere (Ferrage et al., 2015; Dabat et al., 2018, 2020). Briefly, the particles are allowed to settle in a square simulation box with periodic conditions along the $x$ and $y$ axes ( $z$ axis pointing upward; Fig. 2a), according to a steepest descent algorithm to reduce the barycenter altitude. A log normal distribution in the dimensions (i.e., basal surface, particle diameter, ratio between thickness and diameter, and ellipticity degree) of the individual particles was obtained from the work of Ferrage et al. (2015) and based on the experimental morphological study of Reinholdt et al. (2013) for the $0.1-0.2 \mu \mathrm{m}$ size fraction of vermiculite from Santa Olalla, Spain, investigated here. During the settling process, the particles are allowed to slide, swivel or rotate with a random amplitude that ranges from zero to a maximum value. A wide range of degree of anisotropy in the particles' orientation is then obtained by tuning the amplitudes of the movements, leading to a variation in the degree of freedom in the 
particle motions (Ferrage et al., 2015). This degree of anisotropy of the particles' orientation was extracted by calculating the average of the second-order Legendre polynomial on the angular distribution of the particle orientations, as follows:

$$
\left\langle P_{2}\right\rangle=\left\langle P_{2}(\cos \theta)\right\rangle=\left\langle 3 \cos ^{2} \theta-1\right\rangle / 2
$$

where $\theta$ is the angle between the normal unit vector of the particle and the $z$ axis of the simulation box (Fig. 4a). This $\left\langle\mathrm{P}_{2}\right\rangle$ order parameter, also referred to as the nematic order $S$ (Dabat et al., 2018; Underwood and Bourg, 2020) or the Hermans parameter $H$ (Hermans and Platzek, 1939), takes the value of 0 for an isotropic organization and 1 when all of the particles are perfectly oriented in the bedding (all normal to the particles aligned with the $z$ axis of the simulation box).

To cover over a large range of anisotropy degrees in particle orientations, 13 particle packings with $\left\langle\mathrm{P}_{2}\right\rangle$ values that varied from 0.03 to 0.96 were generated according to this algorithm ((Ferrage et al., 2018; Dabat et al., 2020); see Table S1 and Fig. 4a). This methodology leads, however, to different interparticle porosity values as a function of $\left\langle P_{2}\right\rangle$ and does not allow obtaining periodic conditions in the $z$ direction. To overcome these drawbacks for the BD simulations, additional treatments, as detailed by Dabat et al. (2020), were applied to the different obtained VPM. Briefly, a cubic sub-volume of 2000 particles was first extracted, and particles were then polygonized considering 12 in-plane vectors plus 2 vectors passing along the normal of the particles. Periodic conditions with the minimum-image convention were applied along the three directors, $\overrightarrow{e_{x}}, \overrightarrow{e_{y}}$, and $\overrightarrow{e_{z}}$, of the simulation box. Based on the obtained sub-volumes, two subsequent treatments were applied to reduce the interparticle porosity of the packings and to reach an interparticle porosity value that is close to that of the water-saturated samples analyzed in this study by PGSE-NMR and through-diffusion experiments, i.e., $\varepsilon_{\text {interp. }} \sim 0.25$ (Dabat et al., 2020). The first treatment consists of allowing each particle to grow along its 14 vectors, with detection and rejection of particle overlapping. The 
second treatment implies the injection of a new particle on the surface of an existing particle 1106 and the subsequent particle growth to fill the porosity. The two processes led to final 1107 interparticle porosity values of approximately 0.27 for packings with $0.03 \leq\left\langle P_{2}\right\rangle \leq 0.85$ and 1108 approximately 0.25 for packings with $\left\langle P_{2}\right\rangle=0.92$ and 0.96 (Table S1; Fig. 4a). Because these 1109 treatments lead to a decrease in the particle sizes and thus an increase in the overall specific 1110 surface areas (SSA) of the sample, a last treatment involved the dilatation of the simulation box 1111 (and thus particle) dimensions. For vermiculite, the final cubic lengths of the VPM are $\sim 1 \mu \mathrm{m}$ 1112 (i.e., between 1.12 and 0.99 for the order parameter $\left\langle P_{2}\right\rangle$ ranging from 0.03 to 0.96 , respectively; 1113 see Table S1) and of the SSA values are $~ 95 \mathrm{~m}^{2} \mathrm{~g}^{-1}$, which is in agreement with the experimental 1114 results from Reinholdt et al. (2013). The total number of particles ranges between $\sim 11000$ and $1115 \sim 7400$, for the more isotropic and anisotropic organizations, respectively (Table S1). 


\section{Table S. 1.}

1126 Characteristics of the 13 virtual porous media used for the Brownian dynamics simulations.

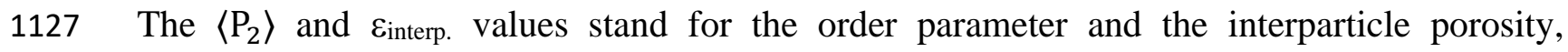

1128 respectively. The $\mathrm{N}_{\text {part. }}$ and $\ell_{\text {box }}$ parameters stand for the total number of particles and the cubic

1129 length of the simulation box (in $\mu \mathrm{m})$, respectively.

\begin{tabular}{ccccc}
\hline Pack. \# & $\left\langle P_{2}\right\rangle$ & $\varepsilon_{\text {interp. }}$ & $\mathrm{N}_{\text {part. }}$ & $\ell_{\text {box }}$ \\
\hline 1 & 0.028 & 0.272 & 10639 & 1.12 \\
2 & 0.053 & 0.274 & 10932 & 1.12 \\
3 & 0.104 & 0.271 & 11039 & 1.13 \\
4 & 0.208 & 0.272 & 11139 & 1.13 \\
5 & 0.322 & 0.272 & 11307 & 1.12 \\
6 & 0.392 & 0.275 & 11092 & 1.12 \\
7 & 0.489 & 0.273 & 11139 & 1.15 \\
8 & 0.575 & 0.275 & 11404 & 1.15 \\
9 & 0.718 & 0.269 & 11446 & 1.16 \\
10 & 0.800 & 0.264 & 11693 & 1.16 \\
11 & 0.849 & 0.264 & 11863 & 1.18 \\
12 & 0.915 & 0.249 & 11526 & 1.18 \\
13 & 0.963 & 0.253 & 7403 & 0.99 \\
\hline
\end{tabular}

1130

\section{References for supplementary data}

1132

1133

1134

1135

Basser, P.J., Mattiello, J., Lebihan, D., 1994. Estimation of the Effective Self-Diffusion Tensor from the NMR Spin Echo. Journal of Magnetic Resonance, Series B 103, 247254. https://doi.org/10.1006/jmrb.1994.1037

Callaghan, P.T., 1991. Principles of nuclear magnetic resonance microscopy. Clarendon Press.

Cotts, R.M., Hoch, M.J.R., Sun, T., Markert, J.T., 1989. Pulsed field gradient stimulated echo methods for improved NMR diffusion measurements in heterogeneous systems. Journal of Magnetic Resonance (1989) 83, 252-266. https://doi.org/10.1016/00222364(89)90189-3

Crank, J., 1975. The Mathematics of Diffusion, 2nd ed. Oxford University Press: London.

Dabat, T., Porion, P., Hubert, F., Paineau, E., Dazas, B., Grégoire, B., Tertre, E., Delville, A., Ferrage, E., 2020. Influence of preferred orientation of clay particles on the diffusion of 
water in kaolinite porous media at constant porosity. Applied Clay Science 184, 105354. https://doi.org/10.1016/j.clay.2019.105354

Dabat, T., Mazurier, A., Hubert, F., Tertre, E., Grégoire, B., Dazas, B., Ferrage, E., 2018. Mesoscale Anisotropy in Porous Media Made of Clay Minerals. A Numerical Study Constrained by Experimental Data. Materials 11, 1972. https://doi.org/10.3390/ma11101972

Ferrage, E., Hubert, F., Tertre, E., Delville, A., Michot, L.J., Levitz, P., 2015. Modeling the arrangement of particles in natural swelling-clay porous media using threedimensional packing of elliptic disks. Physical Review E 91, 062210. https://doi.org/10.1103/PhysRevE.91.062210

Ferrage, E., Hubert, F., Baronnet, A., Grauby, O., Tertre, E., Delville, A., Bihannic, I., Prêt, D., Michot, L.J., Levitz, P., 2018. Influence of crystal structure defects on the smallangle neutron scattering/diffraction patterns of clay-rich porous media. J Appl Crystallogr 51, 1311-1322. https://doi.org/10.1107/S160057671801052X

Melkior, T., 2000. Etude méthodologique de la diffusion de cations interagissant dans des argiles : application : mise en œuvre expérimentale et modélisation du couplage chimie-diffusion d'alcalins dans une bentonite synthétique (Ph.D. Thesis). Ecole Centrale Paris, Paris, France.

Hermans, P.H., Platzek, P., 1939. Beiträge zur Kenntnis des Deformationsmechanismus und der Feinstruktur der Hydratzellulose. Kolloid-Z, Kolloid-Z 88, 68-72.

Porion, P., Ferrage, E., Hubert, F., Tertre, E., Dabat, T., Faugère, A.M., Condé, F., Warmont, F., Delville, A., 2018. Water Mobility within Compacted Clay Samples: Multi-Scale Analysis Exploiting ${ }^{1}$ H NMR Pulsed Gradient Spin Echo and Magnetic Resonance Imaging of Water Density Profiles. ACS Omega 3, 7399-7406. https://doi.org/10.1021/acsomega.8b01083

Reinholdt, M.X., Hubert, F., Faurel, M., Tertre, E., Razafitianamaharavo, A., Francius, G., Prêt, D., Petit, S., Béré, E., Pelletier, M., Ferrage, E., 2013. Morphological properties of vermiculite particles in size-selected fractions obtained by sonication. Applied Clay Science 77-78, 18-32. https://doi.org/10.1016/j.clay.2013.03.013

Sammaljärvi, J., Jokelainen, L., Ikonen, J., Siitari-Kauppi, M., 2012. Free radical polymerisation of MMA with thermal initiator in brick and Grimsel granodiorite. Engineering Geology 135-136, 52-59. https://doi.org/10.1016/j.enggeo.2012.03.005

Savoye, S., Beaucaire, C., Grenut, B., Fayette, A., 2015. Impact of the solution ionic strength on strontium diffusion through the Callovo-Oxfordian clayrocks: An experimental and modeling study. Applied Geochemistry 61, 41-52. https://doi.org/10.1016/j.apgeochem.2015.05.011

Skare, S., Hedehus, M., Moseley, M.E., Li, T.-Q., 2000. Condition Number as a Measure of Noise Performance of Diffusion Tensor Data Acquisition Schemes with MRI. Journal of Magnetic Resonance 147, 340-352. https://doi.org/10.1006/jmre.2000.2209

Stejskal, E.O., Tanner, J.E., 1965. Spin Diffusion Measurements: Spin Echoes in the Presence of a Time-Dependent Field Gradient. The Journal of Chemical Physics 42, 288-292. https://doi.org/10.1063/1.1695690 